methylguanidinium tetraphenylborate, see: Tiritiris (2013b).

\section{Crystal structure of $\mathrm{N}$-[3-(dimethyl- amino)propyl]- $N^{\prime}, N^{\prime}, N^{\prime \prime}, N^{\prime \prime}$-tetramethyl- $N-\left(N, N, N^{\prime}, N^{\prime}\right.$-tetramethylformamidin- iumyl)guanidinium bis(tetraphenyl- borate)}

\section{Ioannis Tiritiris and Willi Kantlehner*}

Fakultät Chemie/Organische Chemie, Hochschule Aalen, Beethovenstrasse 1, D73430 Aalen, Germany. *Correspondence e-mail: willi.kantlehner@hs-aalen.de

Received 23 October 2015; accepted 4 December 2015

Edited by M. Nieger, University of Helsinki, Finland

In the title salt, $\mathrm{C}_{15} \mathrm{H}_{36} \mathrm{~N}_{6}{ }^{2+} \cdot 2 \mathrm{C}_{24} \mathrm{H}_{20} \mathrm{~B}^{-}$, the three $\mathrm{N}-\mathrm{C}$ bond lengths in the central $\mathrm{C}_{3} \mathrm{~N}$ unit of the bisamidinium ion range between 1.388 (3) and 1.506 (3) $\AA$, indicating single- and double-bond character. Furthermore, four $\mathrm{C}-\mathrm{N}$ bonds have double-bond character. Here, the bond lengths range from 1.319 (3) to 1.333 (3) A. Delocalization of the positive charges occurs in the $\mathrm{N} / \mathrm{C} / \mathrm{N}$ and $\mathrm{C} / \mathrm{N} / \mathrm{C}$ planes. The dihedral angle between both $\mathrm{N} / \mathrm{C} / \mathrm{N}$ planes is $70.5(2)^{\circ}$. In the crystal, $\mathrm{C}-$ $\mathrm{H} \cdots \pi$ interactions between $\mathrm{H}$ atoms of the cation and the benzene rings of both tetraphenylborate ions are present. The benzene rings form aromatic pockets, in which the bisamidinium ion is embedded. This leads to the formation of a two-dimensional supramolecular pattern along the $a b$ plane.

Keywords: crystal structure; bisamidinium salt; tetraphenylborate; C$\mathrm{H} \cdots \pi$ interactions.

CCDC reference: 822198

\section{Related literature}

For the synthesis of similar salts to the title compound, see: Bauer et al. (1968). For the crystal structure of $N, N, N^{\prime}, N^{\prime}$ tetramethylchloroformamidinium chloride, see: Tiritiris \& Kantlehner (2008). For the crystal structures of alkali metal tetraphenylborates, see: Behrens et al. (2012a). For the synthesis of $N^{\prime \prime}$-[3-(dimethylamino)propyl]- $N, N, N^{\prime}, N^{\prime}$-tetramethylguanidine, see: Tiritiris \& Kantlehner (2012b). For the crystal structure of $N, N, N^{\prime}, N^{\prime}$-tetramethyl- $N^{\prime \prime}$-[3-(trimethylazaniumyl)propyl]guanidinium bis(tetraphenylborate) acetone disolvate, see: Tiritiris $(2013 a)$. For the crystal structure of $N$-[3-(dimethylamino)propyl]- $N, N^{\prime}, N^{\prime}, N^{\prime \prime}, N^{\prime \prime}$-penta-
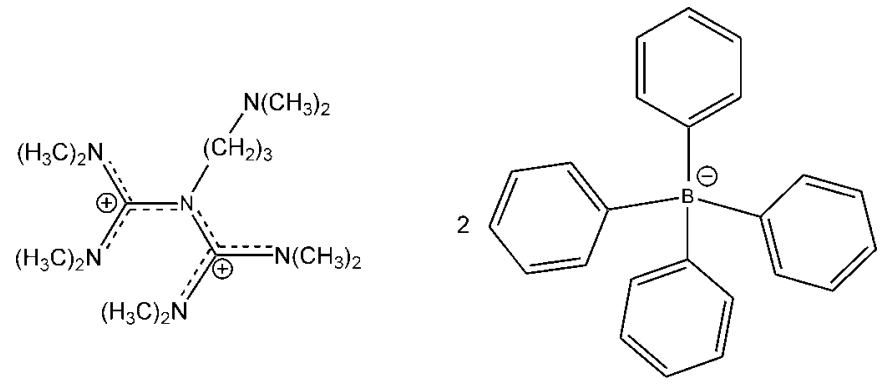

\section{Experimental}

2.1. Crystal data

$\mathrm{C}_{15} \mathrm{H}_{36} \mathrm{~N}_{6}{ }^{2+} \cdot 2 \mathrm{C}_{24} \mathrm{H}_{20} \mathrm{~B}^{-}$

$M_{r}=938.92$

Monoclinic, $C c$

$a=17.1964(5) \AA$

$b=17.6641(7) \AA$

$c=17.4751(6) \AA$

$\beta=98.752(1)^{\circ}$

\subsection{Data collection}

Bruker-Nonius KappaCCD diffractometer

11667 measured reflections

$$
\begin{aligned}
& V=5246.4(3) \AA^{3} \\
& Z=4 \\
& \text { Mo } K \alpha \text { radiation } \\
& \mu=0.07 \mathrm{~mm}^{-1} \\
& T=100 \mathrm{~K} \\
& 0.22 \times 0.18 \times 0.15 \mathrm{~mm}
\end{aligned}
$$

\subsection{Refinement}

$R\left[F^{2}>2 \sigma\left(F^{2}\right)\right]=0.038$

$w R\left(F^{2}\right)=0.087$

$S=1.06$

11660 reflections

651 parameters

2 restraints

$\mathrm{H}$-atom parameters constrained

$\Delta \rho_{\max }=0.21 \mathrm{e}^{-3}$

11660 independent reflections 10531 reflections with $I>2 \sigma(I)$ $R_{\text {int }}=0.040$

\section{Table 1}

Hydrogen-bond geometry $\left(\AA{ }^{\circ}\right)$.

$\mathrm{Cg} 1, \mathrm{Cg} 2$ and $\mathrm{Cg} 3$ are the centroids of the $\mathrm{C} 22-\mathrm{C} 27, \mathrm{C} 28-\mathrm{C} 33$ and $\mathrm{C} 58-\mathrm{C} 63$ rings, respectively.

\begin{tabular}{lllll}
\hline$D-\mathrm{H} \cdots A$ & $D-\mathrm{H}$ & $\mathrm{H} \cdots A$ & $D \cdots A$ & $D-\mathrm{H} \cdots A$ \\
\hline $\mathrm{C} 4-\mathrm{H} 4 B \cdots C g 1$ & 0.98 & 2.63 & $3.597(2)$ & 171 \\
$\mathrm{C} 4-\mathrm{H} 4 A \cdots C g 3$ & 0.98 & 2.64 & $3.580(2)$ & 161 \\
$\mathrm{C} 11-\mathrm{H} 11 A \cdots C g 2$ & 0.99 & 2.94 & $3.500(2)$ & 117 \\
$\mathrm{C} 13-\mathrm{H} 13 B \cdots C g 3$ & 0.99 & 2.97 & $3.950(2)$ & 170 \\
\hline
\end{tabular}

Data collection: COLLECT (Hooft, 2004); cell refinement: DENZO$S M N$ (Otwinowski \& Minor, 1997); data reduction: DENZO-SMN; program(s) used to solve structure: SHELXS97 (Sheldrick, 2008); program(s) used to refine structure: SHELXL2014 (Sheldrick, 2015); molecular graphics: DIAMOND (Brandenburg \& Putz, 2005); software used to prepare material for publication: SHELXL2014. 


\section{Acknowledgements}

The authors thank Dr F. Lissner (Institut für Anorganische Chemie, Universität Stuttgart) for measuring the diffraction data.

Supporting information for this paper is available from the IUCr electronic archives (Reference: NR2062).

\section{References}

Bauer, V. J., Fulmor, W., Morton, G. O. \& Safir, S. R. (1968). J. Am. Chem. Soc. 90, 6845-6846.
Behrens, U., Hoffmann, F. \& Olbrich, F. (2012a). Organometallics, 31, 905-913. Brandenburg, K. \& Putz, H. (2005). DIAMOND. Crystal Impact GbR, Bonn, Germany.

Hooft, R. W. W. (2004). COLLECT. Bruker-Nonius BV, Delft, The Netherlands.

Otwinowski, Z. \& Minor, W. (1997). Methods in Enzymology, Vol. 276, Macromolecular Crystallography, Part A, edited by C. W. Carter Jr \& R. M. Sweet, pp. 307-326. New York: Academic Press.

Parsons, S., Flack, H. D. \& Wagner, T. (2013). Acta Cryst. B69, 249-259.

Sheldrick, G. M. (2008). Acta Cryst. A64, 112-122.

Sheldrick, G. M. (2015). Acta Cryst. C71, 3-8.

Tiritiris, I. (2013a). Acta Cryst. E69, o337-o338.

Tiritiris, I. (2013b). Acta Cryst. E69, o1040.

Tiritiris, I. \& Kantlehner, W. (2008). Z. Kristallogr. 223, 345-346.

Tiritiris, I. \& Kantlehner, W. (2012b). Z. Naturforsch. Teil B, 67, 685-698. 


\section{supporting information}

Acta Cryst. (2015). E71, o1045-o1046 [https://doi.org/10.1107/S2056989015023336]

\section{Crystal structure of $N$-[3-(dimethylamino) propyl]- $N^{\prime}, N^{\prime}, N^{\prime \prime}, N^{\prime \prime}$-tetramethyl-} $N-\left(N, N, N^{\prime}, N^{\prime}\right.$-tetramethylformamidiniumyl)guanidinium bis(tetraphenylborate)

\section{Ioannis Tiritiris and Willi Kantlehner}

\section{S1. Comment}

$\omega$-Aminoalkylguanidines like $N^{\prime \prime}$-[3-(dimethylamino)propyl]- $N, N, N^{\prime}, N^{\prime}$-tetramethylguanidine (I) (Tiritiris \& Kantlehner, $2012 b$ ), in which two nitrogen atoms with different basicity are present, are considered as ambident nucleophiles. Electrophiles can attack at both, on the imine nitrogen of the guanidine function, as well as on the nitrogen atom of the (dimethylamino)propyl group. By reaction of (I) with one equivalent $N, N, N^{\prime}, N^{\prime}$ - tetramethylchloroformamidinium chloride (Tiritiris \& Kantlehner, 2008), the hygroscopic bisamidinium dichloride (II) was formed exclusively. As expected, the reaction occurred preferably at the imine nitrogen of the guanidine function because it is the most basic site. Two similar bisamidinium dichlorides are known in the literature. They where obtained by reaction of $N, N, N^{\prime}, N^{\prime}$ - tetramethylchloroformamidinium chloride (Tiritiris \& Kantlehner, 2008) with $N, N, N^{\prime}, N^{\prime}, N^{\prime \prime}$-pentamethylguanidine or $N$ phenyl- $N^{\prime}, N^{\prime}, N^{\prime \prime}, N^{\prime \prime}$-tetramethylguanidine, respectively (Bauer et al., 1968). In our case, by anion exchange with sodium tetraphenylborate it was possible to obtain a stable, non-hygroscopic salt. The here presented crystal structure is the first structural study of a dicationic nonasubstituted bisamidinium salt. The asymmetric unit contains one twofold charged cation and two tetraphenylborate ions (Fig. 1). Prominent bond parameters in the bisamidinium ion are: N5-C6 = 1.388 (3) $\AA, \mathrm{N} 5-\mathrm{C} 1=1.407$ (3) $\AA, \mathrm{N} 5-\mathrm{C} 11=1.506$ (3) $\AA$, indicating N-C single- and double-bond character of the central $\mathrm{C}_{3} \mathrm{~N}$ unit. The $\mathrm{C}-\mathrm{N}-\mathrm{C}$ angles are $117.51(17)^{\circ}, 118.92(17)^{\circ}$ and $123.16(17)^{\circ}$, indicating a nearly ideal trigonalplanar surrounding of the central nitrogen atom $\mathrm{N} 5$ by the carbon atoms $\mathrm{C} 1, \mathrm{C} 6$ and $\mathrm{C} 11$. The carbon atoms $\mathrm{C} 1$ and $\mathrm{C} 6$ are further surrounded by the nitrogen atoms $\mathrm{N} 1, \mathrm{~N} 2, \mathrm{~N} 3$ and $\mathrm{N} 4$. Here, the $\mathrm{C}-\mathrm{N}$ bonds show double-bond character and the bond lengths range from 1.319 (3) $\AA$ to 1.333 (3) $\AA$. The $\mathrm{N}-\mathrm{C}-\mathrm{N}$ angles range from 118.24 (19) ${ }^{\circ}$ to 121.87 (19) , indicating again nearly ideal trigonal-planar surroundings of both carbon centres by the nitrogen atoms. The positive charges are delocalized in the planes $\mathrm{N} 1 / \mathrm{C} 1 / \mathrm{N} 2, \mathrm{~N} 3 / \mathrm{C} 6 / \mathrm{N} 4$ and $\mathrm{C} 1 / \mathrm{N} 5 / \mathrm{C} 6$. The dihedral angle between the plane $\mathrm{N} 1 / \mathrm{C} 1 / \mathrm{N} 2$ and $\mathrm{N} 3 / \mathrm{C} 6 / \mathrm{N} 4$ is $70.5(2)^{\circ}$. The bond lengths and angles in both tetraphenylborate ions are in good agreement with the data from the crystal structure analysis of the alkali metal tetraphenylborates (Behrens et al., 2012a). C-H $\cdots \pi$ interactions between the bisamidinium hydrogen atoms of $-\mathrm{N}\left(\mathrm{CH}_{3}\right)_{2}$ and $-\mathrm{CH}_{2}$ groups and the phenyl carbon atoms [centroids: $\mathrm{Cg} 1=\mathrm{C} 22-\mathrm{C} 27, \mathrm{Cg} 2=\mathrm{C} 28-\mathrm{C} 33$ and $\mathrm{Cg} 3=\mathrm{C} 58-\mathrm{C} 63$ ] of the tetraphenylborate ion are present (Fig. 2), ranging from 2.63 to $2.97 \AA$ (Tab. 1). Such type of $\mathrm{C}-\mathrm{H} \cdots \pi$ interactions have been observed in tetraphenylborate salts with pentasubstituted or hexasubstituted guanidinium ions [for example: $N, N, N^{\prime}, N^{\prime}$ - tetramethyl- $N^{\prime \prime}$-[3-(trimethylazaniumyl)propyl]guanidinium bis(tetraphenylborate) acetone disolvate (Tiritiris, 2013a); $N$-[3-(Dimethylamino)propyl]$N, N^{\prime}, N^{\prime}, N^{\prime \prime}, N^{\prime \prime}$ - pentamethylguanidinium tetraphenylborate (Tiritiris, 2013b)]. The phenyl rings form aromatic pockets, in which the cation is embedded. This leads finally to the formation of a two-dimensional supramolecular pattern along the $a b$ plane. 


\section{S2. Experimental}

The title compound has been obtained by reaction of $N^{\prime \prime}$-[3-(dimethylamino)propyl]- $N, N, N^{\prime}, N^{\prime}$-tetramethylguanidine (I) (Tiritiris \& Kantlehner, 2012b) with one equivalent of $N, N, N^{\prime}, N^{\prime}$ - tetramethylchloroformamidinium chloride (Tiritiris \& Kantlehner, 2008) in acetonitrile at room temperature. After evaporation of the solvent the crude $N$-[3-(dimethylamino)propyl]- $N$-( $N, N, N^{\prime}, N^{\prime}$-tetramethyl-formamidinio)- $N^{\prime}, N^{\prime}, N^{\prime \prime}, N^{\prime \prime}$-tetramethyl-guanidinium dichloride (II) was washed with diethylether and dried in vacuo. $1.0 \mathrm{~g}$ (2.7 mmol) of (II) was dissolved in $20 \mathrm{ml}$ acetonitrile and $1.83 \mathrm{~g}$ (5.4 mmol) of sodium tetraphenylborate in $20 \mathrm{ml}$ acetonitrile were added. After stirring for one hour at room temperature, the precipitated sodium chloride was filtered off. The title compound crystallized from a saturated acetonitrile solution after a few months at $273 \mathrm{~K}$, forming colorless single crystals. Yield: $2.15 \mathrm{~g}(84.9 \%)$.

\section{S3. Refinement}

A total number of 34135 reflections have been measured. The data where scaled in the chiral point group $C 2$ per default by using SCALEPACK (Otwinowski \& Minor, 1997). After merging all symmetry related reflections and Friedel pairs, a total number of 6569 unique reflections remained (Theta range: $0.41^{\circ}-28.28^{\circ}$; data completeness: $97.7 \%$; $\mathrm{R}_{\mathrm{int}}=0.044$ ). Therefore $\mathrm{R}_{\text {int }}$ given by SHELXL2014/7 (Sheldrick, 2015) is meaningless. The data in the hkl file used for structure solution and refinement were also scaled in the chiral point group $C 2$, but here the Friedel pairs were kept separate. The title compound crystallizes in the non-centrosymmetric space group $C c$; however, in the absence of significant anomalous scattering effects, the determined Flack parameter $\mathrm{x}=-0.8$ (8) (Parsons et al., 2013) is essentially meaningless. The hydrogen atoms of the methyl groups were allowed to rotate with a fixed angle around the $\mathrm{C}-\mathrm{N}$ bond to best fit the experimental electron density, with $U_{\text {iso }}(\mathrm{H})$ set to $1.5 U_{\text {eq }}(\mathrm{C})$ and $\mathrm{d}(\mathrm{C}-\mathrm{H})=0.98 \AA$. The remaining $\mathrm{H}$ atoms were placed in calculated positions with $\mathrm{d}(\mathrm{C}-\mathrm{H})=0.99 \AA$ ( $\mathrm{H}$ atoms in $\mathrm{CH}_{2}$ groups $)$ and $(\mathrm{C}-\mathrm{H})=0.95 \AA$ ( $\mathrm{H}$ atoms in aromatic rings) and refined using a riding model, with $U_{\text {iso }}(\mathrm{H})$ set to $1.2 U_{\text {eq }}(\mathrm{C})$. 


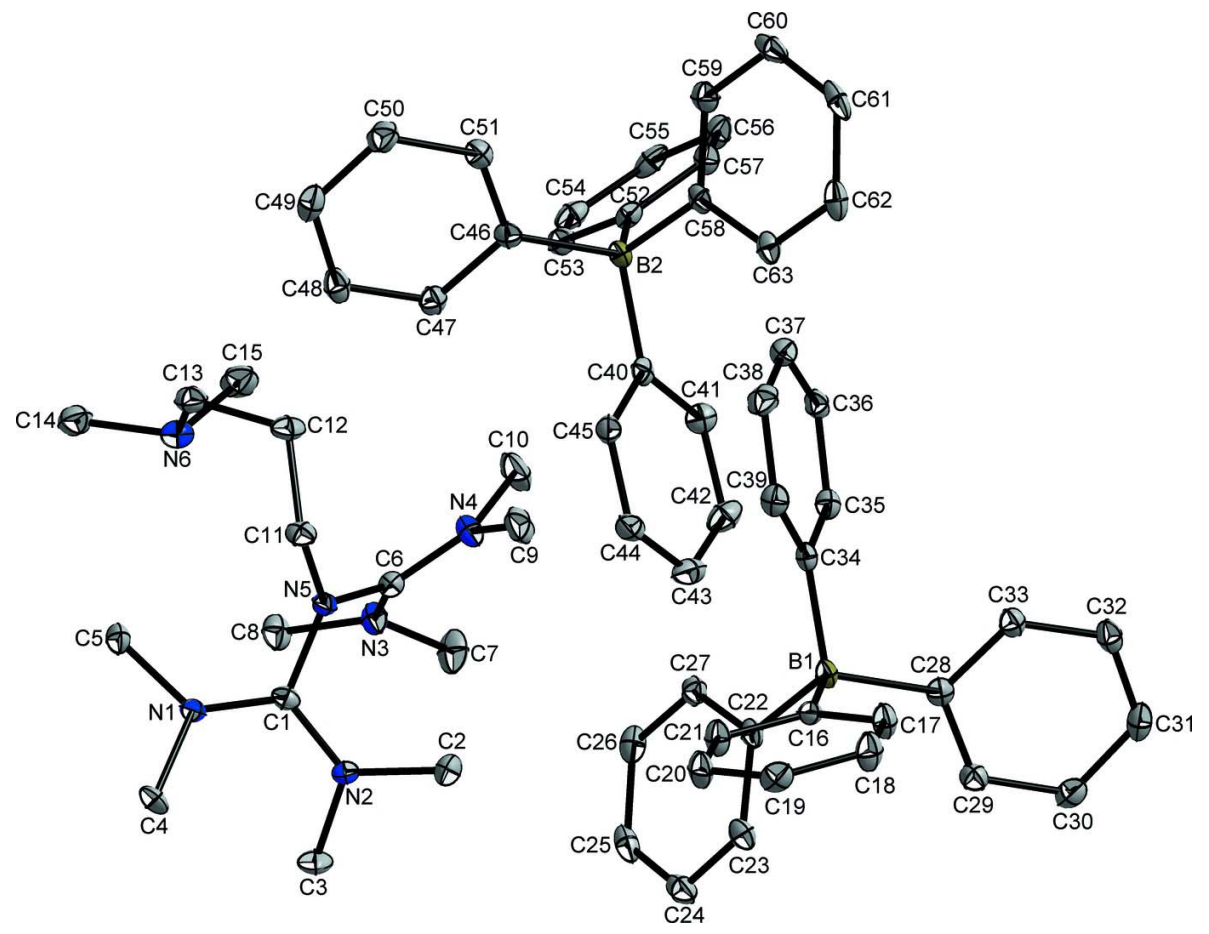

\section{Figure 1}

The structure of the title compound with displacement ellipsoids at the $50 \%$ probability level. All carbon bonded hydrogen atoms were omitted for the sake of clarity.
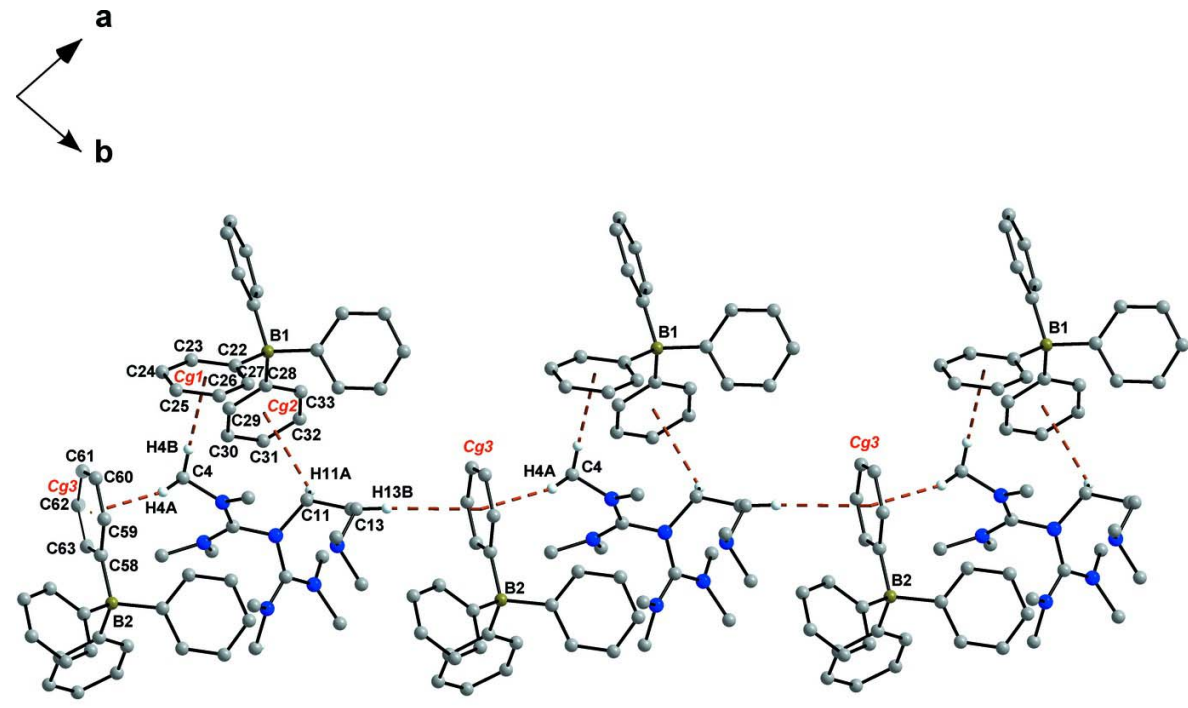

Figure 2

$\mathrm{C}-\mathrm{H} \cdots \pi$ interactions (brown dashed lines) between the hydrogen atoms of the guanidinium ion and the phenyl rings (centroids) of the tetraphenylborate ions. 
$N$-[3-(Dimethylamino) propyl]- $N^{\prime}, N^{\prime}, N^{\prime \prime}, N^{\prime \prime}$-tetramethyl- $N-\left(N, N, N^{\prime}, N^{\prime}-\right.$

tetramethylformamidiniumyl)guanidinium bis(tetraphenylborate)

Crystal data

$\mathrm{C}_{15} \mathrm{H}_{36} \mathrm{~N}_{6}{ }^{2+} \cdot 2 \mathrm{C}_{24} \mathrm{H}_{20} \mathrm{~B}^{-}$

$M_{r}=938.92$

Monoclinic, $C c$

$a=17.1964(5) \AA$

$b=17.6641(7) \AA$

$c=17.4751(6) \AA$

$\beta=98.752(1)^{\circ}$

$V=5246.4(3) \AA^{3}$

$Z=4$

\section{Data collection}

Bruker-Nonius KappaCCD diffractometer

Radiation source: fine-focus sealed tube

Graphite monochromator

$\omega$ scans

11667 measured reflections

11660 independent reflections

Refinement

Refinement on $F^{2}$

Least-squares matrix: full

$R\left[F^{2}>2 \sigma\left(F^{2}\right)\right]=0.038$

$w R\left(F^{2}\right)=0.087$

$S=1.06$

11660 reflections

651 parameters

2 restraints

Primary atom site location: structure-invariant direct methods

Secondary atom site location: difference Fourier map

Hydrogen site location: inferred from neighbouring sites
$F(000)=2024$

$D_{\mathrm{x}}=1.189 \mathrm{Mg} \mathrm{m}^{-3}$

Mo $K \alpha$ radiation, $\lambda=0.71073 \AA$

Cell parameters from 6372 reflections

$\theta=0.4-28.3^{\circ}$

$\mu=0.07 \mathrm{~mm}^{-1}$

$T=100 \mathrm{~K}$

Block, colorless

$0.22 \times 0.18 \times 0.15 \mathrm{~mm}$

10531 reflections with $I>2 \sigma(I)$

$R_{\text {int }}=0.040$

$\theta_{\max }=28.2^{\circ}, \theta_{\min }=3.0^{\circ}$

$h=-22 \rightarrow 22$

$k=-22 \rightarrow 23$

$l=-23 \rightarrow 23$

$\mathrm{H}$-atom parameters constrained

$w=1 /\left[\sigma^{2}\left(F_{\mathrm{o}}^{2}\right)+(0.0318 P)^{2}+3.2124 P\right]$

where $P=\left(F_{\mathrm{o}}{ }^{2}+2 F_{\mathrm{c}}{ }^{2}\right) / 3$

$(\Delta / \sigma)_{\max }<0.001$

$\Delta \rho_{\max }=0.21$ e $\AA^{-3}$

$\Delta \rho_{\min }=-0.20$ e $\AA^{-3}$

Extinction correction: SHELXL2014 (Sheldrick, 2015), $\mathrm{Fc}^{*}=\mathrm{kFc}\left[1+0.001 \mathrm{xFc}^{2} \lambda^{3} / \sin (2 \theta)\right]^{-1 / 4}$

Extinction coefficient: 0.0028 (4)

Absolute structure: Flack $x$ determined using 4294 quotients $\left[\left(I^{+}\right)-(I)\right] /\left[\left(I^{+}\right)+\left(I^{-}\right)\right]$(Parsons et al., 2013)

Absolute structure parameter: $-0.8(8)$

\section{Special details}

Geometry. All esds (except the esd in the dihedral angle between two 1.s. planes) are estimated using the full covariance matrix. The cell esds are taken into account individually in the estimation of esds in distances, angles and torsion angles; correlations between esds in cell parameters are only used when they are defined by crystal symmetry. An approximate (isotropic) treatment of cell esds is used for estimating esds involving l.s. planes.

Refinement. Refinement of $\mathrm{F}^{2}$ against ALL reflections. The weighted R-factor $\mathrm{wR}$ and goodness of fit $\mathrm{S}$ are based on $\mathrm{F}^{2}$, conventional R-factors $R$ are based on $F$, with $F$ set to zero for negative $F^{2}$. The threshold expression of $F^{2}>2 \operatorname{sigma}\left(\mathrm{F}^{2}\right)$ is used only for calculating R-factors(gt) etc. and is not relevant to the choice of reflections for refinement. R-factors based on $\mathrm{F}^{2}$ are statistically about twice as large as those based on F, and R- factors based on ALL data will be even larger.

Fractional atomic coordinates and isotropic or equivalent isotropic displacement parameters $\left(\AA^{2}\right)$

\begin{tabular}{lllll}
\hline & $x$ & $y$ & $z$ & $U_{\text {iso }} * / U_{\text {eq }}$ \\
\hline N1 & $0.54916(10)$ & $0.40299(10)$ & $0.24976(10)$ & $0.0140(4)$
\end{tabular}




\begin{tabular}{|c|c|c|c|c|}
\hline N2 & $0.45346(11)$ & $0.34306(10)$ & $0.30784(11)$ & $0.0159(4)$ \\
\hline N3 & $0.45873(11)$ & $0.21150(10)$ & $0.19896(11)$ & $0.0166(4)$ \\
\hline N4 & 0.53339 (11) & $0.14313(11)$ & $0.29688(11)$ & $0.0188(4)$ \\
\hline N5 & $0.56111(10)$ & $0.27259(10)$ & $0.27842(10)$ & $0.0126(3)$ \\
\hline N6 & $0.65631(11)$ & $0.24425(11)$ & $0.12663(11)$ & $0.0174(4)$ \\
\hline $\mathrm{C} 1$ & $0.52041(12)$ & $0.34173(12)$ & $0.27826(12)$ & $0.0134(4)$ \\
\hline $\mathrm{C} 2$ & $0.43782(14)$ & $0.29283(14)$ & $0.37052(14)$ & $0.0216(5)$ \\
\hline $\mathrm{H} 2 \mathrm{~A}$ & 0.4096 & 0.2478 & 0.3484 & $0.032 *$ \\
\hline H2B & 0.4057 & 0.3196 & 0.4036 & $0.032 *$ \\
\hline $\mathrm{H} 2 \mathrm{C}$ & 0.4877 & 0.2775 & 0.4014 & $0.032 *$ \\
\hline C3 & $0.38691(14)$ & $0.39186(14)$ & $0.27671(15)$ & $0.0241(5)$ \\
\hline $\mathrm{H} 3 \mathrm{~A}$ & 0.3968 & 0.4146 & 0.2280 & $0.036^{*}$ \\
\hline H3B & 0.3808 & 0.4320 & 0.3141 & $0.036^{*}$ \\
\hline $\mathrm{H} 3 \mathrm{C}$ & 0.3387 & 0.3616 & 0.2673 & $0.036^{*}$ \\
\hline $\mathrm{C} 4$ & 0.52999 (14) & 0.47987 (12) & $0.27348(14)$ & $0.0203(5)$ \\
\hline $\mathrm{H} 4 \mathrm{~A}$ & 0.4923 & 0.5032 & 0.2325 & $0.030^{*}$ \\
\hline H4B & 0.5781 & 0.5104 & 0.2824 & $0.030^{*}$ \\
\hline $\mathrm{H} 4 \mathrm{C}$ & 0.5068 & 0.4772 & 0.3213 & $0.030^{*}$ \\
\hline C5 & $0.60081(13)$ & $0.40206(13)$ & 0.19034 (13) & 0.0177 (4) \\
\hline H5A & 0.6084 & 0.3497 & 0.1744 & $0.027^{*}$ \\
\hline H5B & 0.6518 & 0.4242 & 0.2114 & $0.027^{*}$ \\
\hline $\mathrm{H} 5 \mathrm{C}$ & 0.5767 & 0.4316 & 0.1455 & $0.027^{*}$ \\
\hline C6 & $0.51696(12)$ & $0.20783(12)$ & $0.25854(12)$ & $0.0141(4)$ \\
\hline C7 & $0.38500(15)$ & $0.16881(15)$ & $0.19791(15)$ & $0.0257(5)$ \\
\hline H7A & 0.3841 & 0.1458 & 0.2488 & $0.038^{*}$ \\
\hline H7B & 0.3817 & 0.1290 & 0.1585 & $0.038^{*}$ \\
\hline $\mathrm{H} 7 \mathrm{C}$ & 0.3401 & 0.2032 & 0.1858 & $0.038^{*}$ \\
\hline $\mathrm{C} 8$ & $0.46021(14)$ & $0.26164(14)$ & 0.13219 (13) & $0.0197(5)$ \\
\hline H8A & 0.4312 & 0.3082 & 0.1394 & $0.030^{*}$ \\
\hline H8B & 0.4356 & 0.2360 & 0.0849 & $0.030^{*}$ \\
\hline $\mathrm{H} 8 \mathrm{C}$ & 0.5148 & 0.2742 & 0.1277 & $0.030^{*}$ \\
\hline C9 & $0.56851(15)$ & 0.13745 (14) & $0.37887(14)$ & $0.0242(5)$ \\
\hline H9A & 0.6212 & 0.1153 & 0.3828 & $0.036^{*}$ \\
\hline H9B & 0.5354 & 0.1052 & 0.4063 & $0.036^{*}$ \\
\hline $\mathrm{H} 9 \mathrm{C}$ & 0.5723 & 0.1880 & 0.4021 & $0.036^{*}$ \\
\hline $\mathrm{C} 10$ & 0.51657 (17) & $0.06897(13)$ & $0.25923(16)$ & $0.0285(6)$ \\
\hline H10A & 0.4707 & 0.0462 & 0.2773 & $0.043 *$ \\
\hline H10B & 0.5622 & 0.0356 & 0.2724 & $0.043^{*}$ \\
\hline $\mathrm{H} 10 \mathrm{C}$ & 0.5057 & 0.0758 & 0.2030 & $0.043^{*}$ \\
\hline C11 & $0.64786(12)$ & $0.27091(13)$ & 0.30879 (13) & $0.0150(4)$ \\
\hline H11A & 0.6544 & 0.2568 & 0.3642 & $0.018^{*}$ \\
\hline H11B & 0.6686 & 0.3229 & 0.3058 & $0.018^{*}$ \\
\hline $\mathrm{C} 12$ & $0.69866(13)$ & $0.21784(13)$ & $0.26843(13)$ & $0.0188(5)$ \\
\hline $\mathrm{H} 12 \mathrm{~A}$ & 0.6704 & 0.1691 & 0.2591 & $0.023^{*}$ \\
\hline H12B & 0.7478 & 0.2077 & 0.3045 & $0.023 *$ \\
\hline $\mathrm{C} 13$ & $0.72112(13)$ & 0.24477 (13) & $0.19158(13)$ & $0.0186(4)$ \\
\hline H13A & 0.7421 & 0.2969 & 0.1984 & $0.022 *$ \\
\hline H13B & 0.7639 & 0.2120 & 0.1783 & $0.022^{*}$ \\
\hline
\end{tabular}




\begin{tabular}{|c|c|c|c|c|}
\hline $\mathrm{C} 14$ & $0.68101(15)$ & $0.28608(14)$ & $0.06149(14)$ & $0.0227(5)$ \\
\hline $\mathrm{H} 14 \mathrm{~A}$ & 0.7288 & 0.2630 & 0.0476 & $0.034 *$ \\
\hline H14B & 0.6917 & 0.3389 & 0.0766 & $0.034^{*}$ \\
\hline $\mathrm{H} 14 \mathrm{C}$ & 0.6390 & 0.2842 & 0.0169 & $0.034 *$ \\
\hline $\mathrm{C} 15$ & $0.63482(15)$ & $0.16667(14)$ & $0.10226(15)$ & $0.0250(5)$ \\
\hline $\mathrm{H} 15 \mathrm{~A}$ & 0.6812 & 0.1403 & 0.0892 & $0.038^{*}$ \\
\hline H15B & 0.5938 & 0.1679 & 0.0567 & $0.038^{*}$ \\
\hline $\mathrm{H} 15 \mathrm{C}$ & 0.6151 & 0.1399 & 0.1445 & $0.038^{*}$ \\
\hline B1 & $0.29633(14)$ & -0.00569 (13) & $0.41254(14)$ & $0.0131(5)$ \\
\hline $\mathrm{C} 16$ & $0.33345(12)$ & $0.05140(12)$ & $0.48320(13)$ & $0.0135(4)$ \\
\hline $\mathrm{C} 17$ & $0.34030(14)$ & $0.03221(13)$ & $0.56111(13)$ & $0.0189(5)$ \\
\hline H17 & 0.3211 & -0.0156 & 0.5746 & $0.023 *$ \\
\hline $\mathrm{C} 18$ & $0.37429(15)$ & $0.08026(13)$ & $0.62042(13)$ & $0.0214(5)$ \\
\hline H18 & 0.3786 & 0.0645 & 0.6729 & $0.026^{*}$ \\
\hline C19 & $0.40168(14)$ & $0.15091(13)$ & $0.60279(13)$ & $0.0187(5)$ \\
\hline H19 & 0.4238 & 0.1844 & 0.6428 & $0.022 *$ \\
\hline $\mathrm{C} 20$ & $0.39638(14)$ & $0.17187(13)$ & $0.52606(13)$ & $0.0204(5)$ \\
\hline $\mathrm{H} 20$ & 0.4155 & 0.2199 & 0.5130 & $0.024^{*}$ \\
\hline $\mathrm{C} 21$ & $0.36316(14)$ & $0.12288(13)$ & $0.46788(13)$ & $0.0190(5)$ \\
\hline $\mathrm{H} 21$ & 0.3605 & 0.1385 & 0.4155 & $0.023 *$ \\
\hline $\mathrm{C} 22$ & $0.24858(13)$ & $0.04641(12)$ & $0.34214(13)$ & $0.0143(4)$ \\
\hline $\mathrm{C} 23$ & $0.19759(13)$ & $0.10448(12)$ & $0.35885(14)$ & $0.0173(4)$ \\
\hline $\mathrm{H} 23$ & 0.1922 & 0.1133 & 0.4114 & $0.021^{*}$ \\
\hline $\mathrm{C} 24$ & $0.15475(14)$ & $0.14951(13)$ & $0.30210(14)$ & $0.0200(5)$ \\
\hline $\mathrm{H} 24$ & 0.1208 & 0.1877 & 0.3162 & $0.024^{*}$ \\
\hline $\mathrm{C} 25$ & $0.16175(13)$ & $0.13849(13)$ & $0.22473(14)$ & $0.0203(5)$ \\
\hline $\mathrm{H} 25$ & 0.1324 & 0.1687 & 0.1856 & $0.024 *$ \\
\hline $\mathrm{C} 26$ & $0.21206(14)$ & $0.08288(14)$ & $0.20540(13)$ & $0.0190(5)$ \\
\hline $\mathrm{H} 26$ & 0.2178 & 0.0751 & 0.1528 & $0.023^{*}$ \\
\hline $\mathrm{C} 27$ & $0.25439(13)$ & $0.03814(12)$ & $0.26329(13)$ & $0.0156(4)$ \\
\hline $\mathrm{H} 27$ & 0.2886 & 0.0004 & 0.2486 & $0.019^{*}$ \\
\hline $\mathrm{C} 28$ & $0.23664(13)$ & $-0.06677(12)$ & $0.44542(12)$ & $0.0142(4)$ \\
\hline $\mathrm{C} 29$ & $0.15612(13)$ & $-0.05335(13)$ & $0.44582(13)$ & $0.0177(5)$ \\
\hline $\mathrm{H} 29$ & 0.1340 & -0.0074 & 0.4241 & $0.021 *$ \\
\hline $\mathrm{C} 30$ & $0.10721(14)$ & $-0.10448(14)$ & $0.47665(14)$ & $0.0210(5)$ \\
\hline $\mathrm{H} 30$ & 0.0531 & -0.0929 & 0.4757 & $0.025^{*}$ \\
\hline $\mathrm{C} 31$ & $0.13767(14)$ & $-0.17220(14)$ & $0.50867(13)$ & $0.0197(5)$ \\
\hline H31 & 0.1047 & -0.2075 & 0.5293 & $0.024 *$ \\
\hline C32 & $0.21682(14)$ & $-0.18749(13)$ & $0.50998(13)$ & $0.0182(5)$ \\
\hline H32 & 0.2386 & -0.2333 & 0.5322 & $0.022^{*}$ \\
\hline $\mathrm{C} 33$ & $0.26451(14)$ & $-0.13592(12)$ & $0.47879(12)$ & $0.0159(4)$ \\
\hline H33 & 0.3185 & -0.1480 & 0.4801 & $0.019 *$ \\
\hline C34 & $0.36745(13)$ & $-0.05447(12)$ & $0.38311(12)$ & $0.0134(4)$ \\
\hline $\mathrm{C} 35$ & $0.34968(13)$ & $-0.11383(13)$ & $0.32968(13)$ & $0.0151(4)$ \\
\hline H35 & 0.2960 & -0.1231 & 0.3096 & $0.018^{*}$ \\
\hline $\mathrm{C} 36$ & $0.40666(13)$ & $-0.15960(12)$ & $0.30481(12)$ & $0.0157(4)$ \\
\hline H36 & 0.3917 & -0.1991 & 0.2686 & $0.019^{*}$ \\
\hline $\mathrm{C} 37$ & $0.48566(13)$ & $-0.14740(13)$ & $0.33310(13)$ & $0.0187(5)$ \\
\hline
\end{tabular}




\begin{tabular}{|c|c|c|c|c|}
\hline $\mathrm{H} 37$ & 0.5251 & -0.1780 & 0.3160 & $0.022 *$ \\
\hline C38 & 0.50604 (14) & $-0.09022(14)$ & $0.38638(14)$ & $0.0204(5)$ \\
\hline H38 & 0.5598 & -0.0815 & 0.4064 & $0.025^{*}$ \\
\hline C39 & $0.44791(13)$ & $-0.04539(13)$ & $0.41076(13)$ & $0.0169(4)$ \\
\hline H39 & 0.4634 & -0.0068 & 0.4479 & $0.020^{*}$ \\
\hline B2 & $0.80503(15)$ & $0.00432(14)$ & $0.52652(14)$ & $0.0128(4)$ \\
\hline $\mathrm{C} 40$ & $0.73194(13)$ & $0.05104(12)$ & $0.55564(11)$ & $0.0134(4)$ \\
\hline $\mathrm{C} 41$ & $0.65402(13)$ & $0.02539(13)$ & $0.54487(13)$ & $0.0168(4)$ \\
\hline H41 & 0.6413 & -0.0197 & 0.5160 & $0.020^{*}$ \\
\hline $\mathrm{C} 42$ & $0.59422(14)$ & $0.06346(15)$ & $0.57499(13)$ & $0.0217(5)$ \\
\hline $\mathrm{H} 42$ & 0.5422 & 0.0438 & 0.5669 & $0.026^{*}$ \\
\hline $\mathrm{C} 43$ & $0.61052(14)$ & $0.12989(14)$ & $0.61668(13)$ & $0.0212(5)$ \\
\hline $\mathrm{H} 43$ & 0.5701 & 0.1561 & 0.6372 & $0.025^{*}$ \\
\hline $\mathrm{C} 44$ & $0.68678(14)$ & $0.15728(13)$ & $0.62787(13)$ & $0.0178(5)$ \\
\hline H44 & 0.6988 & 0.2030 & 0.6557 & $0.021 *$ \\
\hline $\mathrm{C} 45$ & $0.74583(13)$ & $0.11816(13)$ & $0.59849(12)$ & $0.0157(4)$ \\
\hline H45 & 0.7979 & 0.1377 & 0.6078 & $0.019^{*}$ \\
\hline $\mathrm{C} 46$ & $0.86800(13)$ & $0.06551(12)$ & $0.49821(12)$ & $0.0138(4)$ \\
\hline $\mathrm{C} 47$ & $0.84134(13)$ & $0.13320(13)$ & $0.45996(12)$ & $0.0160(4)$ \\
\hline H47 & 0.7866 & 0.1440 & 0.4528 & $0.019^{*}$ \\
\hline $\mathrm{C} 48$ & $0.89142(14)$ & $0.18477(13)$ & $0.43229(13)$ & $0.0185(5)$ \\
\hline H48 & 0.8706 & 0.2297 & 0.4072 & $0.022^{*}$ \\
\hline C49 & $0.97173(15)$ & $0.17088(14)$ & $0.44126(14)$ & $0.0206(5)$ \\
\hline H49 & 1.0063 & 0.2057 & 0.4221 & $0.025^{*}$ \\
\hline $\mathrm{C} 50$ & $1.00057(14)$ & $0.10506(14)$ & $0.47875(14)$ & $0.0214(5)$ \\
\hline $\mathrm{H} 50$ & 1.0553 & 0.0945 & 0.4854 & $0.026^{*}$ \\
\hline $\mathrm{C} 51$ & $0.94928(13)$ & $0.05445(13)$ & $0.50663(13)$ & $0.0174(4)$ \\
\hline H51 & 0.9707 & 0.0102 & 0.5327 & $0.021^{*}$ \\
\hline $\mathrm{C} 52$ & $0.76945(12)$ & $-0.04893(13)$ & $0.45171(12)$ & $0.0141(4)$ \\
\hline C53 & $0.73887(13)$ & $-0.01733(13)$ & $0.37920(12)$ & $0.0165(4)$ \\
\hline H53 & 0.7429 & 0.0358 & 0.3723 & $0.020^{*}$ \\
\hline $\mathrm{C} 54$ & $0.70306(13)$ & $-0.06078(14)$ & $0.31746(13)$ & $0.0187(5)$ \\
\hline H54 & 0.6831 & -0.0371 & 0.2697 & $0.022^{*}$ \\
\hline C55 & $0.69626(13)$ & $-0.13857(14)$ & $0.32521(13)$ & $0.0195(5)$ \\
\hline H55 & 0.6709 & -0.1683 & 0.2834 & $0.023^{*}$ \\
\hline $\mathrm{C} 56$ & $0.72690(14)$ & $-0.17218(13)$ & $0.39466(13)$ & $0.0188(5)$ \\
\hline H56 & 0.7237 & -0.2255 & 0.4005 & $0.023^{*}$ \\
\hline $\mathrm{C} 57$ & $0.76263(13)$ & $-0.12778(13)$ & $0.45626(13)$ & $0.0165(4)$ \\
\hline H57 & 0.7833 & -0.1522 & 0.5034 & $0.020^{*}$ \\
\hline $\mathrm{C} 58$ & $0.84769(12)$ & $-0.04918(12)$ & $0.59834(13)$ & $0.0133(4)$ \\
\hline C59 & $0.90731(13)$ & $-0.10152(13)$ & $0.58859(14)$ & $0.0171(4)$ \\
\hline H59 & 0.9228 & -0.1061 & 0.5389 & $0.021^{*}$ \\
\hline $\mathrm{C} 60$ & $0.94459(13)$ & $-0.14677(13)$ & $0.64832(14)$ & $0.0195(5)$ \\
\hline H60 & 0.9848 & -0.1809 & 0.6389 & $0.023 *$ \\
\hline C61 & $0.92316(14)$ & $-0.14212(12)$ & $0.72185(14)$ & $0.0202(5)$ \\
\hline H61 & 0.9489 & -0.1724 & 0.7630 & $0.024 *$ \\
\hline C62 & $0.86401(14)$ & $-0.09282(13)$ & $0.73399(13)$ & $0.0192(5)$ \\
\hline H62 & 0.8482 & -0.0895 & 0.7837 & $0.023^{*}$ \\
\hline
\end{tabular}




\begin{tabular}{lllll} 
C63 & $0.82717(13)$ & $-0.04758(12)$ & $0.67306(13)$ & $0.0151(4)$ \\
H63 & 0.7864 & -0.0143 & 0.6828 & $0.018^{*}$ \\
\hline
\end{tabular}

Atomic displacement parameters $\left(\AA^{2}\right)$

\begin{tabular}{|c|c|c|c|c|c|c|}
\hline & $U^{11}$ & $U^{22}$ & $U^{33}$ & $U^{12}$ & $U^{13}$ & $U^{23}$ \\
\hline N1 & $0.0133(8)$ & $0.0109(8)$ & $0.0181(9)$ & $0.0019(7)$ & $0.0032(7)$ & $-0.0004(7)$ \\
\hline $\mathrm{N} 2$ & $0.0132(9)$ & $0.0157(9)$ & $0.0196(9)$ & $0.0029(7)$ & $0.0056(7)$ & $0.0028(7)$ \\
\hline N3 & $0.0143(9)$ & $0.0160(9)$ & $0.0187(9)$ & $-0.0027(7)$ & $-0.0005(7)$ & $0.0019(7)$ \\
\hline N4 & $0.0214(10)$ & $0.0139(9)$ & $0.0207(10)$ & $0.0006(7)$ & $0.0022(8)$ & $0.0039(7)$ \\
\hline N5 & $0.0113(8)$ & $0.0114(8)$ & $0.0148(8)$ & $0.0014(7)$ & $0.0012(7)$ & $0.0012(7)$ \\
\hline N6 & $0.0162(9)$ & $0.0182(9)$ & $0.0173(9)$ & $0.0019(7)$ & $0.0015(7)$ & $-0.0021(7)$ \\
\hline $\mathrm{C} 1$ & $0.0131(10)$ & $0.0132(10)$ & $0.0134(10)$ & $0.0012(8)$ & $-0.0003(8)$ & $-0.0013(8)$ \\
\hline $\mathrm{C} 2$ & $0.0234(12)$ & $0.0217(12)$ & $0.0223(12)$ & $0.0010(9)$ & $0.0115(10)$ & $0.0040(9)$ \\
\hline $\mathrm{C} 3$ & $0.0144(11)$ & $0.0249(12)$ & 0.0337 (13) & $0.0080(9)$ & $0.0056(9)$ & $0.0059(10)$ \\
\hline $\mathrm{C} 4$ & $0.0251(12)$ & $0.0089(10)$ & $0.0277(12)$ & $0.0014(9)$ & $0.0067(10)$ & $-0.0007(9)$ \\
\hline $\mathrm{C} 5$ & $0.0194(11)$ & $0.0149(10)$ & $0.0200(11)$ & $-0.0027(8)$ & $0.0067(9)$ & $0.0018(8)$ \\
\hline C6 & $0.0144(10)$ & $0.0137(10)$ & $0.0151(10)$ & $0.0005(8)$ & $0.0046(8)$ & $0.0006(8)$ \\
\hline $\mathrm{C} 7$ & $0.0198(12)$ & $0.0272(13)$ & $0.0287(12)$ & $-0.0119(10)$ & $-0.0005(10)$ & $0.0024(10)$ \\
\hline $\mathrm{C} 8$ & $0.0184(11)$ & $0.0228(12)$ & $0.0164(11)$ & $-0.0007(9)$ & $-0.0018(9)$ & $0.0051(9)$ \\
\hline C9 & $0.0275(13)$ & $0.0219(12)$ & $0.0224(12)$ & $0.0030(10)$ & $0.0014(10)$ & $0.0095(9)$ \\
\hline $\mathrm{C} 10$ & $0.0381(15)$ & $0.0126(11)$ & $0.0346(14)$ & $-0.0021(10)$ & $0.0053(11)$ & $0.0016(10)$ \\
\hline $\mathrm{C} 11$ & $0.0111(10)$ & $0.0161(11)$ & $0.0169(10)$ & $0.0007(8)$ & $-0.0013(8)$ & $-0.0001(8)$ \\
\hline $\mathrm{C} 12$ & $0.0143(10)$ & $0.0204(11)$ & 0.0209 (11) & $0.0062(8)$ & $0.0006(8)$ & 0.0007 (9) \\
\hline $\mathrm{C} 13$ & $0.0146(10)$ & $0.0191(11)$ & $0.0222(11)$ & $0.0027(8)$ & $0.0028(9)$ & $-0.0009(9)$ \\
\hline $\mathrm{C} 14$ & $0.0238(12)$ & $0.0250(12)$ & $0.0203(11)$ & $0.0043(10)$ & $0.0067(9)$ & $-0.0008(9)$ \\
\hline $\mathrm{C} 15$ & $0.0259(13)$ & $0.0240(12)$ & $0.0263(12)$ & $-0.0019(10)$ & $0.0074(10)$ & $-0.0071(10)$ \\
\hline B1 & $0.0155(12)$ & $0.0106(11)$ & $0.0130(11)$ & $-0.0014(9)$ & $0.0015(9)$ & $0.0010(9)$ \\
\hline $\mathrm{C} 16$ & $0.0121(10)$ & $0.0123(10)$ & $0.0164(10)$ & $0.0017(8)$ & $0.0031(8)$ & $-0.0006(8)$ \\
\hline $\mathrm{C} 17$ & $0.0255(12)$ & $0.0142(10)$ & $0.0173(11)$ & $-0.0049(9)$ & $0.0040(9)$ & $0.0006(9)$ \\
\hline $\mathrm{C} 18$ & $0.0300(13)$ & $0.0219(12)$ & $0.0127(10)$ & $-0.0053(10)$ & $0.0042(9)$ & $0.0001(9)$ \\
\hline C19 & $0.0199(11)$ & $0.0189(11)$ & $0.0179(11)$ & $-0.0038(9)$ & $0.0042(9)$ & $-0.0071(9)$ \\
\hline $\mathrm{C} 20$ & $0.0240(12)$ & $0.0149(11)$ & $0.0226(12)$ & $-0.0072(9)$ & $0.0043(9)$ & $0.0003(9)$ \\
\hline $\mathrm{C} 21$ & $0.0240(12)$ & $0.0186(11)$ & $0.0144(10)$ & $-0.0044(9)$ & $0.0033(9)$ & $0.0009(8)$ \\
\hline $\mathrm{C} 22$ & $0.0147(10)$ & $0.0114(10)$ & $0.0166(10)$ & $-0.0042(8)$ & $0.0020(8)$ & $0.0009(8)$ \\
\hline $\mathrm{C} 23$ & 0.0198 (11) & $0.0118(10)$ & $0.0201(11)$ & $-0.0026(8)$ & $0.0022(9)$ & $-0.0005(8)$ \\
\hline $\mathrm{C} 24$ & $0.0179(11)$ & $0.0123(11)$ & $0.0291(12)$ & $-0.0011(9)$ & $0.0010(9)$ & $0.0012(9)$ \\
\hline $\mathrm{C} 25$ & $0.0167(11)$ & $0.0171(11)$ & $0.0249(12)$ & $-0.0049(9)$ & $-0.0041(9)$ & $0.0096(9)$ \\
\hline $\mathrm{C} 26$ & $0.0188(11)$ & $0.0226(12)$ & $0.0154(10)$ & $-0.0057(9)$ & $0.0017(8)$ & $0.0039(9)$ \\
\hline $\mathrm{C} 27$ & $0.0148(10)$ & $0.0142(10)$ & $0.0177(11)$ & $-0.0029(8)$ & $0.0025(8)$ & $0.0009(8)$ \\
\hline $\mathrm{C} 28$ & $0.0180(11)$ & $0.0131(10)$ & $0.0114(10)$ & $-0.0012(8)$ & $0.0015(8)$ & $-0.0017(8)$ \\
\hline $\mathrm{C} 29$ & $0.0208(11)$ & $0.0148(11)$ & $0.0181(11)$ & $0.0002(9)$ & $0.0050(9)$ & $-0.0002(8)$ \\
\hline $\mathrm{C} 30$ & $0.0175(11)$ & $0.0241(12)$ & $0.0227(12)$ & $-0.0019(9)$ & $0.0077(9)$ & $-0.0034(9)$ \\
\hline $\mathrm{C} 31$ & $0.0270(12)$ & $0.0188(11)$ & $0.0145(10)$ & $-0.0087(9)$ & $0.0067(9)$ & $-0.0014(9)$ \\
\hline $\mathrm{C} 32$ & $0.0278(12)$ & $0.0136(11)$ & $0.0135(10)$ & $-0.0019(9)$ & $0.0043(9)$ & $0.0003(8)$ \\
\hline $\mathrm{C} 33$ & $0.0185(11)$ & $0.0141(11)$ & $0.0152(10)$ & $-0.0005(8)$ & $0.0030(8)$ & $0.0003(8)$ \\
\hline C34 & $0.0161(10)$ & $0.0120(10)$ & $0.0122(10)$ & $-0.0005(8)$ & $0.0027(8)$ & $0.0034(8)$ \\
\hline C35 & $0.0149(10)$ & $0.0154(11)$ & $0.0149(10)$ & $-0.0023(8)$ & $0.0020(8)$ & $0.0017(8)$ \\
\hline
\end{tabular}




\begin{tabular}{|c|c|c|c|c|c|c|}
\hline C36 & $0.0222(11)$ & $0.0127(10)$ & $0.0125(10)$ & $0.0002(8)$ & $0.0040(8)$ & $0.0018(8)$ \\
\hline $\mathrm{C} 37$ & $0.0194(11)$ & $0.0186(11)$ & $0.0196(11)$ & $0.0039(9)$ & $0.0077(9)$ & $0.0042(9)$ \\
\hline $\mathrm{C} 38$ & $0.0143(10)$ & $0.0250(12)$ & $0.0221(11)$ & $-0.0005(9)$ & $0.0029(8)$ & $0.0029(9)$ \\
\hline C39 & 0.0185 (11) & $0.0170(11)$ & $0.0151(10)$ & -0.0028 & $0.0023(8)$ & $-0.0008(8)$ \\
\hline B2 & $0.0141(11)$ & $0.0102(11)$ & $0.0140(11)$ & $-0.0010(9)$ & $0.0019(9)$ & $0.0002(9)$ \\
\hline C40 & $0.0169(10)$ & $0.0146(10)$ & $0.0083(9)$ & $0.0010(8)$ & $0.0014(8)$ & $0.0033(8)$ \\
\hline $\mathrm{C} 41$ & $0.0165(11)$ & $0.0192(11)$ & $0.0140(10)$ & $0.0006(9)$ & $0.0005(8)$ & $-0.0011(8)$ \\
\hline $\mathrm{C} 42$ & $0.0144(11)$ & $0.0321(13)$ & $0.0186(11)$ & $0.0000(9)$ & $0.0020(9)$ & $-0.0005(10)$ \\
\hline C43 & $0.0204(12)$ & $0.0287(13)$ & $0.0154(11)$ & $0.0097(10)$ & $0.0053(9)$ & $0.0022(9)$ \\
\hline C44 & $0.0260(12)$ & $0.0152(11)$ & $0.0126(10)$ & $0.0039(9)$ & $0.0045(9)$ & $0.0001(8)$ \\
\hline $\mathrm{C} 45$ & $0.0183(11)$ & $0.0149(11)$ & $0.0143(10)$ & $-0.0014(8)$ & $0.0042(8)$ & $0.0013(8)$ \\
\hline $\mathrm{C} 46$ & $0.0163(10)$ & $0.0124(10)$ & $0.0128(10)$ & $-0.0012(8)$ & $0.0023(8)$ & $-0.0028(8)$ \\
\hline C47 & $0.0176(11)$ & $0.0165(11)$ & $0.0137(10)$ & $-0.0002(8)$ & $0.0024(8)$ & $-0.0009(8)$ \\
\hline C48 & $0.0267(12)$ & $0.0158(11)$ & $0.0128(10)$ & $-0.0023(9)$ & $0.0022(9)$ & $0.0008(8)$ \\
\hline C49 & $0.0240(12)$ & $0.0208(12)$ & $0.0185(11)$ & $-0.0080(9)$ & $0.0082(9)$ & $-0.0012(9)$ \\
\hline $\mathrm{C} 50$ & $0.0172(11)$ & $0.0218(12)$ & $0.0262(12)$ & $-0.0030(9)$ & $0.0062(9)$ & $-0.0020(10)$ \\
\hline C51 & $0.0183(11)$ & $0.0140(10)$ & $0.0199(11)$ & 0.0008 (8) & $0.0032(9)$ & $0.0004(8)$ \\
\hline C52 & $0.0116(10)$ & $0.0172(11)$ & $0.0145(10)$ & $-0.0017(8)$ & $0.0046(8)$ & $-0.0016(8)$ \\
\hline C53 & $0.0143(10)$ & $0.0190(11)$ & $0.0167(11)$ & $0.0006(8)$ & $0.0038(8)$ & $0.0006(9)$ \\
\hline C54 & $0.0143(10)$ & $0.0290(13)$ & $0.0128(10)$ & $0.0013(9)$ & $0.0025(8)$ & $-0.0011(9)$ \\
\hline C55 & $0.0151(11)$ & $0.0294(13)$ & $0.0152(10)$ & $-0.0055(9)$ & $0.0059(8)$ & $-0.0080(9)$ \\
\hline C56 & $0.0207(11)$ & $0.0181(11)$ & $0.0189(11)$ & $-0.0063(9)$ & $0.0070(9)$ & $-0.0044(9)$ \\
\hline C57 & $0.0187(11)$ & $0.0169(11)$ & $0.0143(10)$ & $-0.0029(8)$ & $0.0041(8)$ & $0.0007(8)$ \\
\hline C58 & $0.0138(10)$ & $0.0089(10)$ & $0.0167(10)$ & $-0.0043(8)$ & $0.0003(8)$ & $-0.0007(8)$ \\
\hline C59 & $0.0169(11)$ & $0.0145(11)$ & $0.0198(11)$ & $-0.0028(9)$ & $0.0020(9)$ & $-0.0022(9)$ \\
\hline C60 & $0.0159(11)$ & $0.0091(10)$ & $0.0319(13)$ & $-0.0014(8)$ & $-0.0013(9)$ & $-0.0003(9)$ \\
\hline C61 & $0.0227(12)$ & $0.0112(10)$ & $0.0235(12)$ & $-0.0068(9)$ & $-0.0072(9)$ & $0.0056(9)$ \\
\hline C62 & $0.0246(12)$ & $0.0165(11)$ & $0.0156(10)$ & $-0.0087(9)$ & $-0.0004(9)$ & $0.0026(9)$ \\
\hline C63 & $0.0157(10)$ & $0.0129(10)$ & $0.0157(10)$ & $-0.0045(8)$ & $-0.0004(8)$ & $0.0003(8)$ \\
\hline
\end{tabular}

Geometric parameters $(\AA, \stackrel{\circ}{)})$

\begin{tabular}{|c|c|c|c|}
\hline $\mathrm{N} 1-\mathrm{C} 1$ & $1.319(3)$ & $\mathrm{C} 24-\mathrm{H} 24$ & 0.9500 \\
\hline $\mathrm{N} 1-\mathrm{C} 5$ & $1.465(3)$ & $\mathrm{C} 25-\mathrm{C} 26$ & $1.384(4)$ \\
\hline $\mathrm{N} 1-\mathrm{C} 4$ & $1.472(3)$ & $\mathrm{C} 25-\mathrm{H} 25$ & 0.9500 \\
\hline $\mathrm{N} 2-\mathrm{C} 1$ & $1.332(3)$ & $\mathrm{C} 26-\mathrm{C} 27$ & $1.398(3)$ \\
\hline $\mathrm{N} 2-\mathrm{C} 2$ & $1.466(3)$ & $\mathrm{C} 26-\mathrm{H} 26$ & 0.9500 \\
\hline $\mathrm{N} 2-\mathrm{C} 3$ & $1.469(3)$ & $\mathrm{C} 27-\mathrm{H} 27$ & 0.9500 \\
\hline N3-C6 & $1.332(3)$ & C28-C29 & $1.406(3)$ \\
\hline $\mathrm{N} 3-\mathrm{C} 8$ & $1.468(3)$ & $\mathrm{C} 28-\mathrm{C} 33$ & $1.406(3)$ \\
\hline $\mathrm{N} 3-\mathrm{C} 7$ & $1.473(3)$ & $\mathrm{C} 29-\mathrm{C} 30$ & $1.397(3)$ \\
\hline $\mathrm{N} 4-\mathrm{C} 6$ & $1.333(3)$ & C29-H29 & 0.9500 \\
\hline $\mathrm{N} 4-\mathrm{C} 9$ & $1.471(3)$ & C30-C31 & $1.389(3)$ \\
\hline $\mathrm{N} 4-\mathrm{C} 10$ & $1.475(3)$ & $\mathrm{C} 30-\mathrm{H} 30$ & 0.9500 \\
\hline N5-C6 & $1.388(3)$ & C31-C32 & $1.384(3)$ \\
\hline $\mathrm{N} 5-\mathrm{C} 1$ & $1.407(3)$ & C31-H31 & 0.9500 \\
\hline N5-C11 & $1.506(3)$ & C32-C33 & $1.391(3)$ \\
\hline N6-C13 & $1.465(3)$ & C32-H32 & 0.9500 \\
\hline
\end{tabular}




\begin{tabular}{|c|c|c|c|}
\hline N6- $\mathrm{C} 15$ & $1.466(3)$ & $\mathrm{C} 33-\mathrm{H} 33$ & 0.9500 \\
\hline N6-C14 & $1.473(3)$ & $\mathrm{C} 34-\mathrm{C} 39$ & $1.404(3)$ \\
\hline $\mathrm{C} 2-\mathrm{H} 2 \mathrm{~A}$ & 0.9800 & $\mathrm{C} 34-\mathrm{C} 35$ & $1.406(3)$ \\
\hline $\mathrm{C} 2-\mathrm{H} 2 \mathrm{~B}$ & 0.9800 & $\mathrm{C} 35-\mathrm{C} 36$ & $1.390(3)$ \\
\hline $\mathrm{C} 2-\mathrm{H} 2 \mathrm{C}$ & 0.9800 & $\mathrm{C} 35-\mathrm{H} 35$ & 0.9500 \\
\hline $\mathrm{C} 3-\mathrm{H} 3 \mathrm{~A}$ & 0.9800 & $\mathrm{C} 36-\mathrm{C} 37$ & $1.390(3)$ \\
\hline $\mathrm{C} 3-\mathrm{H} 3 \mathrm{~B}$ & 0.9800 & C36-H36 & 0.9500 \\
\hline $\mathrm{C} 3-\mathrm{H} 3 \mathrm{C}$ & 0.9800 & $\mathrm{C} 37-\mathrm{C} 38$ & $1.382(3)$ \\
\hline $\mathrm{C} 4-\mathrm{H} 4 \mathrm{~A}$ & 0.9800 & C37-H37 & 0.9500 \\
\hline $\mathrm{C} 4-\mathrm{H} 4 \mathrm{~B}$ & 0.9800 & $\mathrm{C} 38-\mathrm{C} 39$ & $1.392(3)$ \\
\hline $\mathrm{C} 4-\mathrm{H} 4 \mathrm{C}$ & 0.9800 & C $38-\mathrm{H} 38$ & 0.9500 \\
\hline C5-H5A & 0.9800 & C39-H39 & 0.9500 \\
\hline C5-H5B & 0.9800 & $\mathrm{~B} 2-\mathrm{C} 40$ & $1.648(3)$ \\
\hline $\mathrm{C} 5-\mathrm{H} 5 \mathrm{C}$ & 0.9800 & $\mathrm{~B} 2-\mathrm{C} 52$ & $1.651(3)$ \\
\hline C7-H7A & 0.9800 & $\mathrm{~B} 2-\mathrm{C} 58$ & $1.651(3)$ \\
\hline $\mathrm{C} 7-\mathrm{H} 7 \mathrm{~B}$ & 0.9800 & $\mathrm{~B} 2-\mathrm{C} 46$ & $1.658(3)$ \\
\hline $\mathrm{C} 7-\mathrm{H} 7 \mathrm{C}$ & 0.9800 & $\mathrm{C} 40-\mathrm{C} 41$ & $1.400(3)$ \\
\hline $\mathrm{C} 8-\mathrm{H} 8 \mathrm{~A}$ & 0.9800 & $\mathrm{C} 40-\mathrm{C} 45$ & $1.403(3)$ \\
\hline $\mathrm{C} 8-\mathrm{H} 8 \mathrm{~B}$ & 0.9800 & $\mathrm{C} 41-\mathrm{C} 42$ & $1.397(3)$ \\
\hline $\mathrm{C} 8-\mathrm{H} 8 \mathrm{C}$ & 0.9800 & $\mathrm{C} 41-\mathrm{H} 41$ & 0.9500 \\
\hline $\mathrm{C} 9-\mathrm{H} 9 \mathrm{~A}$ & 0.9800 & $\mathrm{C} 42-\mathrm{C} 43$ & $1.387(3)$ \\
\hline C9-H9B & 0.9800 & $\mathrm{C} 42-\mathrm{H} 42$ & 0.9500 \\
\hline $\mathrm{C} 9-\mathrm{H} 9 \mathrm{C}$ & 0.9800 & $\mathrm{C} 43-\mathrm{C} 44$ & $1.384(3)$ \\
\hline $\mathrm{C} 10-\mathrm{H} 10 \mathrm{~A}$ & 0.9800 & $\mathrm{C} 43-\mathrm{H} 43$ & 0.9500 \\
\hline $\mathrm{C} 10-\mathrm{H} 10 \mathrm{~B}$ & 0.9800 & $\mathrm{C} 44-\mathrm{C} 45$ & $1.390(3)$ \\
\hline $\mathrm{C} 10-\mathrm{H} 10 \mathrm{C}$ & 0.9800 & $\mathrm{C} 44-\mathrm{H} 44$ & 0.9500 \\
\hline $\mathrm{C} 11-\mathrm{C} 12$ & $1.525(3)$ & $\mathrm{C} 45-\mathrm{H} 45$ & 0.9500 \\
\hline $\mathrm{C} 11-\mathrm{H} 11 \mathrm{~A}$ & 0.9900 & $\mathrm{C} 46-\mathrm{C} 51$ & $1.397(3)$ \\
\hline $\mathrm{C} 11-\mathrm{H} 11 \mathrm{~B}$ & 0.9900 & $\mathrm{C} 46-\mathrm{C} 47$ & $1.412(3)$ \\
\hline $\mathrm{C} 12-\mathrm{C} 13$ & $1.529(3)$ & $\mathrm{C} 47-\mathrm{C} 48$ & $1.390(3)$ \\
\hline $\mathrm{C} 12-\mathrm{H} 12 \mathrm{~A}$ & 0.9900 & $\mathrm{C} 47-\mathrm{H} 47$ & 0.9500 \\
\hline $\mathrm{C} 12-\mathrm{H} 12 \mathrm{~B}$ & 0.9900 & $\mathrm{C} 48-\mathrm{C} 49$ & $1.388(4)$ \\
\hline $\mathrm{C} 13-\mathrm{H} 13 \mathrm{~A}$ & 0.9900 & $\mathrm{C} 48-\mathrm{H} 48$ & 0.9500 \\
\hline C13-H13B & 0.9900 & $\mathrm{C} 49-\mathrm{C} 50$ & $1.389(4)$ \\
\hline $\mathrm{C} 14-\mathrm{H} 14 \mathrm{~A}$ & 0.9800 & $\mathrm{C} 49-\mathrm{H} 49$ & 0.9500 \\
\hline C14-H14B & 0.9800 & $\mathrm{C} 50-\mathrm{C} 51$ & $1.395(3)$ \\
\hline $\mathrm{C} 14-\mathrm{H} 14 \mathrm{C}$ & 0.9800 & $\mathrm{C} 50-\mathrm{H} 50$ & 0.9500 \\
\hline $\mathrm{C} 15-\mathrm{H} 15 \mathrm{~A}$ & 0.9800 & $\mathrm{C} 51-\mathrm{H} 51$ & 0.9500 \\
\hline $\mathrm{C} 15-\mathrm{H} 15 \mathrm{~B}$ & 0.9800 & $\mathrm{C} 52-\mathrm{C} 57$ & $1.401(3)$ \\
\hline $\mathrm{C} 15-\mathrm{H} 15 \mathrm{C}$ & 0.9800 & $\mathrm{C} 52-\mathrm{C} 53$ & $1.411(3)$ \\
\hline $\mathrm{B} 1-\mathrm{C} 34$ & $1.641(3)$ & $\mathrm{C} 53-\mathrm{C} 54$ & $1.389(3)$ \\
\hline $\mathrm{B} 1-\mathrm{C} 16$ & $1.646(3)$ & $\mathrm{C} 53-\mathrm{H} 53$ & 0.9500 \\
\hline $\mathrm{B} 1-\mathrm{C} 28$ & $1.651(3)$ & $\mathrm{C} 54-\mathrm{C} 55$ & $1.387(3)$ \\
\hline $\mathrm{B} 1-\mathrm{C} 22$ & $1.651(3)$ & C54-H54 & 0.9500 \\
\hline $\mathrm{C} 16-\mathrm{C} 17$ & $1.390(3)$ & $\mathrm{C} 55-\mathrm{C} 56$ & $1.381(3)$ \\
\hline $\mathrm{C} 16-\mathrm{C} 21$ & $1.403(3)$ & $\mathrm{C} 55-\mathrm{H} 55$ & 0.9500 \\
\hline $\mathrm{C} 17-\mathrm{C} 18$ & $1.397(3)$ & $\mathrm{C} 56-\mathrm{C} 57$ & $1.397(3)$ \\
\hline $\mathrm{C} 17-\mathrm{H} 17$ & 0.9500 & $\mathrm{C} 56-\mathrm{H} 56$ & 0.9500 \\
\hline
\end{tabular}




\begin{tabular}{|c|c|c|c|}
\hline $\mathrm{C} 18-\mathrm{C} 19$ & $1.385(3)$ & C57-H57 & 0.9500 \\
\hline C18-H18 & 0.9500 & $\mathrm{C} 58-\mathrm{C} 63$ & $1.404(3)$ \\
\hline $\mathrm{C} 19-\mathrm{C} 20$ & $1.381(3)$ & $\mathrm{C} 58-\mathrm{C} 59$ & $1.410(3)$ \\
\hline C19-H19 & 0.9500 & $\mathrm{C} 59-\mathrm{C} 60$ & $1.391(3)$ \\
\hline $\mathrm{C} 20-\mathrm{C} 21$ & $1.390(3)$ & C59-H59 & 0.9500 \\
\hline $\mathrm{C} 20-\mathrm{H} 20$ & 0.9500 & $\mathrm{C} 60-\mathrm{C} 61$ & $1.392(4)$ \\
\hline $\mathrm{C} 21-\mathrm{H} 21$ & 0.9500 & $\mathrm{C} 60-\mathrm{H} 60$ & 0.9500 \\
\hline $\mathrm{C} 22-\mathrm{C} 27$ & $1.404(3)$ & C61-C62 & $1.380(4)$ \\
\hline $\mathrm{C} 22-\mathrm{C} 23$ & $1.409(3)$ & C61-H61 & 0.9500 \\
\hline $\mathrm{C} 23-\mathrm{C} 24$ & $1.392(3)$ & C62-C63 & $1.403(3)$ \\
\hline $\mathrm{C} 23-\mathrm{H} 23$ & 0.9500 & C62-H62 & 0.9500 \\
\hline $\mathrm{C} 24-\mathrm{C} 25$ & $1.389(4)$ & C63-H63 & 0.9500 \\
\hline $\mathrm{C} 1-\mathrm{N} 1-\mathrm{C} 5$ & $124.12(18)$ & $\mathrm{C} 24-\mathrm{C} 23-\mathrm{C} 22$ & $123.2(2)$ \\
\hline $\mathrm{C} 1-\mathrm{N} 1-\mathrm{C} 4$ & $122.54(18)$ & $\mathrm{C} 24-\mathrm{C} 23-\mathrm{H} 23$ & 118.4 \\
\hline $\mathrm{C} 5-\mathrm{N} 1-\mathrm{C} 4$ & $113.31(18)$ & $\mathrm{C} 22-\mathrm{C} 23-\mathrm{H} 23$ & 118.4 \\
\hline $\mathrm{C} 1-\mathrm{N} 2-\mathrm{C} 2$ & $123.16(19)$ & $\mathrm{C} 25-\mathrm{C} 24-\mathrm{C} 23$ & $119.9(2)$ \\
\hline $\mathrm{C} 1-\mathrm{N} 2-\mathrm{C} 3$ & $122.49(19)$ & $\mathrm{C} 25-\mathrm{C} 24-\mathrm{H} 24$ & 120.0 \\
\hline $\mathrm{C} 2-\mathrm{N} 2-\mathrm{C} 3$ & $114.24(19)$ & $\mathrm{C} 23-\mathrm{C} 24-\mathrm{H} 24$ & 120.0 \\
\hline $\mathrm{C} 6-\mathrm{N} 3-\mathrm{C} 8$ & $123.28(18)$ & $\mathrm{C} 26-\mathrm{C} 25-\mathrm{C} 24$ & $119.1(2)$ \\
\hline $\mathrm{C} 6-\mathrm{N} 3-\mathrm{C} 7$ & $121.89(19)$ & $\mathrm{C} 26-\mathrm{C} 25-\mathrm{H} 25$ & 120.4 \\
\hline $\mathrm{C} 8-\mathrm{N} 3-\mathrm{C} 7$ & $114.71(18)$ & $\mathrm{C} 24-\mathrm{C} 25-\mathrm{H} 25$ & 120.4 \\
\hline $\mathrm{C} 6-\mathrm{N} 4-\mathrm{C} 9$ & $124.90(19)$ & $\mathrm{C} 25-\mathrm{C} 26-\mathrm{C} 27$ & $120.0(2)$ \\
\hline $\mathrm{C} 6-\mathrm{N} 4-\mathrm{C} 10$ & $121.67(19)$ & $\mathrm{C} 25-\mathrm{C} 26-\mathrm{H} 26$ & 120.0 \\
\hline $\mathrm{C} 9-\mathrm{N} 4-\mathrm{C} 10$ & $113.42(19)$ & $\mathrm{C} 27-\mathrm{C} 26-\mathrm{H} 26$ & 120.0 \\
\hline $\mathrm{C} 6-\mathrm{N} 5-\mathrm{C} 1$ & $117.51(17)$ & $\mathrm{C} 26-\mathrm{C} 27-\mathrm{C} 22$ & $123.0(2)$ \\
\hline $\mathrm{C} 6-\mathrm{N} 5-\mathrm{C} 11$ & $123.16(17)$ & $\mathrm{C} 26-\mathrm{C} 27-\mathrm{H} 27$ & 118.5 \\
\hline $\mathrm{C} 1-\mathrm{N} 5-\mathrm{C} 11$ & $118.92(17)$ & $\mathrm{C} 22-\mathrm{C} 27-\mathrm{H} 27$ & 118.5 \\
\hline $\mathrm{C} 13-\mathrm{N} 6-\mathrm{C} 15$ & $111.06(18)$ & $\mathrm{C} 29-\mathrm{C} 28-\mathrm{C} 33$ & $114.7(2)$ \\
\hline $\mathrm{C} 13-\mathrm{N} 6-\mathrm{C} 14$ & $108.72(18)$ & $\mathrm{C} 29-\mathrm{C} 28-\mathrm{B} 1$ & $123.8(2)$ \\
\hline $\mathrm{C} 15-\mathrm{N} 6-\mathrm{C} 14$ & $109.52(18)$ & $\mathrm{C} 33-\mathrm{C} 28-\mathrm{B} 1$ & $121.42(19)$ \\
\hline $\mathrm{N} 1-\mathrm{C} 1-\mathrm{N} 2$ & $121.87(19)$ & $\mathrm{C} 30-\mathrm{C} 29-\mathrm{C} 28$ & $123.0(2)$ \\
\hline $\mathrm{N} 1-\mathrm{C} 1-\mathrm{N} 5$ & $119.87(19)$ & $\mathrm{C} 30-\mathrm{C} 29-\mathrm{H} 29$ & 118.5 \\
\hline $\mathrm{N} 2-\mathrm{C} 1-\mathrm{N} 5$ & $118.26(19)$ & $\mathrm{C} 28-\mathrm{C} 29-\mathrm{H} 29$ & 118.5 \\
\hline $\mathrm{N} 2-\mathrm{C} 2-\mathrm{H} 2 \mathrm{~A}$ & 109.5 & $\mathrm{C} 31-\mathrm{C} 30-\mathrm{C} 29$ & $120.0(2)$ \\
\hline $\mathrm{N} 2-\mathrm{C} 2-\mathrm{H} 2 \mathrm{~B}$ & 109.5 & $\mathrm{C} 31-\mathrm{C} 30-\mathrm{H} 30$ & 120.0 \\
\hline $\mathrm{H} 2 \mathrm{~A}-\mathrm{C} 2-\mathrm{H} 2 \mathrm{~B}$ & 109.5 & $\mathrm{C} 29-\mathrm{C} 30-\mathrm{H} 30$ & 120.0 \\
\hline $\mathrm{N} 2-\mathrm{C} 2-\mathrm{H} 2 \mathrm{C}$ & 109.5 & $\mathrm{C} 32-\mathrm{C} 31-\mathrm{C} 30$ & $119.0(2)$ \\
\hline $\mathrm{H} 2 \mathrm{~A}-\mathrm{C} 2-\mathrm{H} 2 \mathrm{C}$ & 109.5 & $\mathrm{C} 32-\mathrm{C} 31-\mathrm{H} 31$ & 120.5 \\
\hline $\mathrm{H} 2 \mathrm{~B}-\mathrm{C} 2-\mathrm{H} 2 \mathrm{C}$ & 109.5 & $\mathrm{C} 30-\mathrm{C} 31-\mathrm{H} 31$ & 120.5 \\
\hline $\mathrm{N} 2-\mathrm{C} 3-\mathrm{H} 3 \mathrm{~A}$ & 109.5 & $\mathrm{C} 31-\mathrm{C} 32-\mathrm{C} 33$ & $120.2(2)$ \\
\hline $\mathrm{N} 2-\mathrm{C} 3-\mathrm{H} 3 \mathrm{~B}$ & 109.5 & $\mathrm{C} 31-\mathrm{C} 32-\mathrm{H} 32$ & 119.9 \\
\hline $\mathrm{H} 3 \mathrm{~A}-\mathrm{C} 3-\mathrm{H} 3 \mathrm{~B}$ & 109.5 & $\mathrm{C} 33-\mathrm{C} 32-\mathrm{H} 32$ & 119.9 \\
\hline $\mathrm{N} 2-\mathrm{C} 3-\mathrm{H} 3 \mathrm{C}$ & 109.5 & $\mathrm{C} 32-\mathrm{C} 33-\mathrm{C} 28$ & $123.2(2)$ \\
\hline $\mathrm{H} 3 \mathrm{~A}-\mathrm{C} 3-\mathrm{H} 3 \mathrm{C}$ & 109.5 & $\mathrm{C} 32-\mathrm{C} 33-\mathrm{H} 33$ & 118.4 \\
\hline $\mathrm{H} 3 \mathrm{~B}-\mathrm{C} 3-\mathrm{H} 3 \mathrm{C}$ & 109.5 & $\mathrm{C} 28-\mathrm{C} 33-\mathrm{H} 33$ & 118.4 \\
\hline $\mathrm{N} 1-\mathrm{C} 4-\mathrm{H} 4 \mathrm{~A}$ & 109.5 & $\mathrm{C} 39-\mathrm{C} 34-\mathrm{C} 35$ & $114.7(2)$ \\
\hline $\mathrm{N} 1-\mathrm{C} 4-\mathrm{H} 4 \mathrm{~B}$ & 109.5 & $\mathrm{C} 39-\mathrm{C} 34-\mathrm{B} 1$ & $125.07(19)$ \\
\hline
\end{tabular}


$\mathrm{H} 4 \mathrm{~A}-\mathrm{C} 4-\mathrm{H} 4 \mathrm{~B}$

$\mathrm{N} 1-\mathrm{C} 4-\mathrm{H} 4 \mathrm{C}$

$\mathrm{H} 4 \mathrm{~A}-\mathrm{C} 4-\mathrm{H} 4 \mathrm{C}$

$\mathrm{H} 4 \mathrm{~B}-\mathrm{C} 4-\mathrm{H} 4 \mathrm{C}$

N1-C5-H5A

$\mathrm{N} 1-\mathrm{C} 5-\mathrm{H} 5 \mathrm{~B}$

$\mathrm{H} 5 \mathrm{~A}-\mathrm{C} 5-\mathrm{H} 5 \mathrm{~B}$

$\mathrm{N} 1-\mathrm{C} 5-\mathrm{H} 5 \mathrm{C}$

$\mathrm{H} 5 \mathrm{~A}-\mathrm{C} 5-\mathrm{H} 5 \mathrm{C}$

$\mathrm{H} 5 \mathrm{~B}-\mathrm{C} 5-\mathrm{H} 5 \mathrm{C}$

$\mathrm{N} 3-\mathrm{C} 6-\mathrm{N} 4$

$\mathrm{N} 3-\mathrm{C} 6-\mathrm{N} 5$

$\mathrm{N} 4-\mathrm{C} 6-\mathrm{N} 5$

N3-C7-H7A

N3-C7-H7B

$\mathrm{H} 7 \mathrm{~A}-\mathrm{C} 7-\mathrm{H} 7 \mathrm{~B}$

N3-C7- $77 \mathrm{C}$

$\mathrm{H} 7 \mathrm{~A}-\mathrm{C} 7-\mathrm{H} 7 \mathrm{C}$

$\mathrm{H} 7 \mathrm{~B}-\mathrm{C} 7-\mathrm{H} 7 \mathrm{C}$

N3- 8 - $\mathrm{H} 8 \mathrm{~A}$

$\mathrm{N} 3-\mathrm{C} 8-\mathrm{H} 8 \mathrm{~B}$

$\mathrm{H} 8 \mathrm{~A}-\mathrm{C} 8-\mathrm{H} 8 \mathrm{~B}$

$\mathrm{N} 3-\mathrm{C} 8-\mathrm{H} 8 \mathrm{C}$

$\mathrm{H} 8 \mathrm{~A}-\mathrm{C} 8-\mathrm{H} 8 \mathrm{C}$

$\mathrm{H} 8 \mathrm{~B}-\mathrm{C} 8-\mathrm{H} 8 \mathrm{C}$

N4-C9- $\mathrm{H} 9 \mathrm{~A}$

N4-C9-H9B

H9A-C9- H9B

N4-C9- $\mathrm{H} 9 \mathrm{C}$

$\mathrm{H} 9 \mathrm{~A}-\mathrm{C} 9-\mathrm{H} 9 \mathrm{C}$

H9B-C9-H9C

N4-C10-H10A

N4-C10-H10B

$\mathrm{H} 10 \mathrm{~A}-\mathrm{C} 10-\mathrm{H} 10 \mathrm{~B}$

$\mathrm{N} 4-\mathrm{C} 10-\mathrm{H} 10 \mathrm{C}$

$\mathrm{H} 10 \mathrm{~A}-\mathrm{C} 10-\mathrm{H} 10 \mathrm{C}$

$\mathrm{H} 10 \mathrm{~B}-\mathrm{C} 10-\mathrm{H} 10 \mathrm{C}$

N5-C11-C12

N5-C11-H11A

C12-C11-H11A

N5-C11-H11B

C12-C11-H11B

H11A-C11-H11B

C11-C12-C13

$\mathrm{C} 11-\mathrm{C} 12-\mathrm{H} 12 \mathrm{~A}$

C13-C12-H12A

C11-C12-H12B

C13-C12-H12B
109.5

109.5

109.5

109.5

109.5

109.5

109.5

109.5

109.5

109.5

120.8 (2)

118.24 (19)

120.99 (19)

109.5

109.5

109.5

109.5

109.5

109.5

109.5

109.5

109.5

109.5

109.5

109.5

109.5

109.5

109.5

109.5

109.5

109.5

109.5

109.5

109.5

109.5

109.5

109.5

117.10 (18)

108.0

108.0

108.0

108.0

107.3

117.00 (19)

108.0

108.0

108.0

108.0
$\mathrm{C} 35-\mathrm{C} 34-\mathrm{B} 1$

$\mathrm{C} 36-\mathrm{C} 35-\mathrm{C} 34$

$\mathrm{C} 36-\mathrm{C} 35-\mathrm{H} 35$

$\mathrm{C} 34-\mathrm{C} 35-\mathrm{H} 35$

C37-C $36-\mathrm{C} 35$

$\mathrm{C} 37-\mathrm{C} 36-\mathrm{H} 36$

$\mathrm{C} 35-\mathrm{C} 36-\mathrm{H} 36$

C $38-\mathrm{C} 37-\mathrm{C} 36$

$\mathrm{C} 38-\mathrm{C} 37-\mathrm{H} 37$

C36- C37- 337

C37-C $38-\mathrm{C} 39$

$\mathrm{C} 37-\mathrm{C} 38-\mathrm{H} 38$

C39- $338-\mathrm{H} 38$

C38-C39-C34

C38-C39-H39

C34-C39- H39

C40-B2-C52

$\mathrm{C} 40-\mathrm{B} 2-\mathrm{C} 58$

$\mathrm{C} 52-\mathrm{B} 2-\mathrm{C} 58$

$\mathrm{C} 40-\mathrm{B} 2-\mathrm{C} 46$

$\mathrm{C} 52-\mathrm{B} 2-\mathrm{C} 46$

$\mathrm{C} 58-\mathrm{B} 2-\mathrm{C} 46$

$\mathrm{C} 41-\mathrm{C} 40-\mathrm{C} 45$

$\mathrm{C} 41-\mathrm{C} 40-\mathrm{B} 2$

$\mathrm{C} 45-\mathrm{C} 40-\mathrm{B} 2$

$\mathrm{C} 42-\mathrm{C} 41-\mathrm{C} 40$

$\mathrm{C} 42-\mathrm{C} 41-\mathrm{H} 41$

$\mathrm{C} 40-\mathrm{C} 41-\mathrm{H} 41$

$\mathrm{C} 43-\mathrm{C} 42-\mathrm{C} 41$

$\mathrm{C} 43-\mathrm{C} 42-\mathrm{H} 42$

$\mathrm{C} 41-\mathrm{C} 42-\mathrm{H} 42$

$\mathrm{C} 44-\mathrm{C} 43-\mathrm{C} 42$

$\mathrm{C} 44-\mathrm{C} 43-\mathrm{H} 43$

$\mathrm{C} 42-\mathrm{C} 43-\mathrm{H} 43$

$\mathrm{C} 43-\mathrm{C} 44-\mathrm{C} 45$

$\mathrm{C} 43-\mathrm{C} 44-\mathrm{H} 44$

$\mathrm{C} 45-\mathrm{C} 44-\mathrm{H} 44$

$\mathrm{C} 44-\mathrm{C} 45-\mathrm{C} 40$

$\mathrm{C} 44-\mathrm{C} 45-\mathrm{H} 45$

$\mathrm{C} 40-\mathrm{C} 45-\mathrm{H} 45$

$\mathrm{C} 51-\mathrm{C} 46-\mathrm{C} 47$

$\mathrm{C} 51-\mathrm{C} 46-\mathrm{B} 2$

$\mathrm{C} 47-\mathrm{C} 46-\mathrm{B} 2$

$\mathrm{C} 48-\mathrm{C} 47-\mathrm{C} 46$

$\mathrm{C} 48-\mathrm{C} 47-\mathrm{H} 47$

$\mathrm{C} 46-\mathrm{C} 47-\mathrm{H} 47$

$\mathrm{C} 49-\mathrm{C} 48-\mathrm{C} 47$

$\mathrm{C} 49-\mathrm{C} 48-\mathrm{H} 48$
120.16 (19)

123.3 (2)

118.4

118.4

119.7 (2)

120.1

120.1

119.1 (2)

120.4

120.4

120.1 (2)

119.9

119.9

123.1 (2)

118.5

118.5

108.77 (18)

108.87 (18)

110.12 (17)

109.23 (17)

108.39 (17)

111.42 (18)

115.3 (2)

123.78 (19)

120.80 (19)

122.5 (2)

118.7

118.7

120.2 (2)

119.9

119.9

118.8 (2)

120.6

120.6

120.3 (2)

119.9

119.9

122.9 (2)

118.6

118.6

114.6 (2)

124.47 (19)

120.90 (19)

123.1 (2)

118.5

118.5

120.2 (2)

119.9 


\begin{tabular}{|c|c|c|c|}
\hline $\mathrm{H} 12 \mathrm{~A}-\mathrm{C} 12-\mathrm{H} 12 \mathrm{~B}$ & 107.3 & $\mathrm{C} 47-\mathrm{C} 48-\mathrm{H} 48$ & 119.9 \\
\hline $\mathrm{N} 6-\mathrm{C} 13-\mathrm{C} 12$ & $114.50(19)$ & $\mathrm{C} 48-\mathrm{C} 49-\mathrm{C} 50$ & $118.6(2)$ \\
\hline $\mathrm{N} 6-\mathrm{C} 13-\mathrm{H} 13 \mathrm{~A}$ & 108.6 & $\mathrm{C} 48-\mathrm{C} 49-\mathrm{H} 49$ & 120.7 \\
\hline $\mathrm{C} 12-\mathrm{C} 13-\mathrm{H} 13 \mathrm{~A}$ & 108.6 & $\mathrm{C} 50-\mathrm{C} 49-\mathrm{H} 49$ & 120.7 \\
\hline $\mathrm{N} 6-\mathrm{C} 13-\mathrm{H} 13 \mathrm{~B}$ & 108.6 & $\mathrm{C} 49-\mathrm{C} 50-\mathrm{C} 51$ & $120.1(2)$ \\
\hline $\mathrm{C} 12-\mathrm{C} 13-\mathrm{H} 13 \mathrm{~B}$ & 108.6 & $\mathrm{C} 49-\mathrm{C} 50-\mathrm{H} 50$ & 119.9 \\
\hline $\mathrm{H} 13 \mathrm{~A}-\mathrm{C} 13-\mathrm{H} 13 \mathrm{~B}$ & 107.6 & $\mathrm{C} 51-\mathrm{C} 50-\mathrm{H} 50$ & 119.9 \\
\hline $\mathrm{N} 6-\mathrm{C} 14-\mathrm{H} 14 \mathrm{~A}$ & 109.5 & $\mathrm{C} 50-\mathrm{C} 51-\mathrm{C} 46$ & $123.3(2)$ \\
\hline $\mathrm{N} 6-\mathrm{C} 14-\mathrm{H} 14 \mathrm{~B}$ & 109.5 & $\mathrm{C} 50-\mathrm{C} 51-\mathrm{H} 51$ & 118.3 \\
\hline $\mathrm{H} 14 \mathrm{~A}-\mathrm{C} 14-\mathrm{H} 14 \mathrm{~B}$ & 109.5 & $\mathrm{C} 46-\mathrm{C} 51-\mathrm{H} 51$ & 118.3 \\
\hline $\mathrm{N} 6-\mathrm{C} 14-\mathrm{H} 14 \mathrm{C}$ & 109.5 & $\mathrm{C} 57-\mathrm{C} 52-\mathrm{C} 53$ & $114.92(19)$ \\
\hline $\mathrm{H} 14 \mathrm{~A}-\mathrm{C} 14-\mathrm{H} 14 \mathrm{C}$ & 109.5 & $\mathrm{C} 57-\mathrm{C} 52-\mathrm{B} 2$ & $123.07(19)$ \\
\hline $\mathrm{H} 14 \mathrm{~B}-\mathrm{C} 14-\mathrm{H} 14 \mathrm{C}$ & 109.5 & $\mathrm{C} 53-\mathrm{C} 52-\mathrm{B} 2$ & $121.89(19)$ \\
\hline $\mathrm{N} 6-\mathrm{C} 15-\mathrm{H} 15 \mathrm{~A}$ & 109.5 & $\mathrm{C} 54-\mathrm{C} 53-\mathrm{C} 52$ & $122.6(2)$ \\
\hline N6-C15-H15B & 109.5 & $\mathrm{C} 54-\mathrm{C} 53-\mathrm{H} 53$ & 118.7 \\
\hline $\mathrm{H} 15 \mathrm{~A}-\mathrm{C} 15-\mathrm{H} 15 \mathrm{~B}$ & 109.5 & $\mathrm{C} 52-\mathrm{C} 53-\mathrm{H} 53$ & 118.7 \\
\hline $\mathrm{N} 6-\mathrm{C} 15-\mathrm{H} 15 \mathrm{C}$ & 109.5 & $\mathrm{C} 55-\mathrm{C} 54-\mathrm{C} 53$ & $120.3(2)$ \\
\hline $\mathrm{H} 15 \mathrm{~A}-\mathrm{C} 15-\mathrm{H} 15 \mathrm{C}$ & 109.5 & $\mathrm{C} 55-\mathrm{C} 54-\mathrm{H} 54$ & 119.8 \\
\hline $\mathrm{H} 15 \mathrm{~B}-\mathrm{C} 15-\mathrm{H} 15 \mathrm{C}$ & 109.5 & $\mathrm{C} 53-\mathrm{C} 54-\mathrm{H} 54$ & 119.8 \\
\hline $\mathrm{C} 34-\mathrm{B} 1-\mathrm{C} 16$ & $109.53(18)$ & $\mathrm{C} 56-\mathrm{C} 55-\mathrm{C} 54$ & $119.0(2)$ \\
\hline $\mathrm{C} 34-\mathrm{B} 1-\mathrm{C} 28$ & $107.35(17)$ & $\mathrm{C} 56-\mathrm{C} 55-\mathrm{H} 55$ & 120.5 \\
\hline $\mathrm{C} 16-\mathrm{B} 1-\mathrm{C} 28$ & $109.26(18)$ & $\mathrm{C} 54-\mathrm{C} 55-\mathrm{H} 55$ & 120.5 \\
\hline $\mathrm{C} 34-\mathrm{B} 1-\mathrm{C} 22$ & $111.64(18)$ & $\mathrm{C} 55-\mathrm{C} 56-\mathrm{C} 57$ & $120.0(2)$ \\
\hline $\mathrm{C} 16-\mathrm{B} 1-\mathrm{C} 22$ & 108.03 & $\mathrm{C} 55-\mathrm{C} 56-\mathrm{H} 56$ & 120.0 \\
\hline $\mathrm{C} 28-\mathrm{B} 1-\mathrm{C} 22$ & $111.00(18)$ & $\mathrm{C} 57-\mathrm{C} 56-\mathrm{H} 56$ & 120.0 \\
\hline $\mathrm{C} 17-\mathrm{C} 16-\mathrm{C} 21$ & $115.3(2)$ & $\mathrm{C} 56-\mathrm{C} 57-\mathrm{C} 52$ & $123.1(2)$ \\
\hline $\mathrm{C} 17-\mathrm{C} 16-\mathrm{B} 1$ & $123.41(19)$ & C56-C57-H57 & 118.5 \\
\hline $\mathrm{C} 21-\mathrm{C} 16-\mathrm{B} 1$ & $121.27(19)$ & $\mathrm{C} 52-\mathrm{C} 57-\mathrm{H} 57$ & 118.5 \\
\hline $\mathrm{C} 16-\mathrm{C} 17-\mathrm{C} 18$ & $122.8(2)$ & $\mathrm{C} 63-\mathrm{C} 58-\mathrm{C} 59$ & $114.6(2)$ \\
\hline $\mathrm{C} 16-\mathrm{C} 17-\mathrm{H} 17$ & 118.6 & $\mathrm{C} 63-\mathrm{C} 58-\mathrm{B} 2$ & $123.37(19)$ \\
\hline $\mathrm{C} 18-\mathrm{C} 17-\mathrm{H} 17$ & 118.6 & $\mathrm{C} 59-\mathrm{C} 58-\mathrm{B} 2$ & $122.0(2)$ \\
\hline $\mathrm{C} 19-\mathrm{C} 18-\mathrm{C} 17$ & $120.0(2)$ & $\mathrm{C} 60-\mathrm{C} 59-\mathrm{C} 58$ & $123.1(2)$ \\
\hline $\mathrm{C} 19-\mathrm{C} 18-\mathrm{H} 18$ & 120.0 & $\mathrm{C} 60-\mathrm{C} 59-\mathrm{H} 59$ & 118.4 \\
\hline $\mathrm{C} 17-\mathrm{C} 18-\mathrm{H} 18$ & 120.0 & $\mathrm{C} 58-\mathrm{C} 59-\mathrm{H} 59$ & 118.4 \\
\hline $\mathrm{C} 20-\mathrm{C} 19-\mathrm{C} 18$ & $118.9(2)$ & $\mathrm{C} 59-\mathrm{C} 60-\mathrm{C} 61$ & $120.1(2)$ \\
\hline $\mathrm{C} 20-\mathrm{C} 19-\mathrm{H} 19$ & 120.6 & $\mathrm{C} 59-\mathrm{C} 60-\mathrm{H} 60$ & 119.9 \\
\hline $\mathrm{C} 18-\mathrm{C} 19-\mathrm{H} 19$ & 120.6 & $\mathrm{C} 61-\mathrm{C} 60-\mathrm{H} 60$ & 119.9 \\
\hline $\mathrm{C} 19-\mathrm{C} 20-\mathrm{C} 21$ & $120.2(2)$ & C62-C61-C60 & $118.9(2)$ \\
\hline $\mathrm{C} 19-\mathrm{C} 20-\mathrm{H} 20$ & 119.9 & $\mathrm{C} 62-\mathrm{C} 61-\mathrm{H} 61$ & 120.5 \\
\hline $\mathrm{C} 21-\mathrm{C} 20-\mathrm{H} 20$ & 119.9 & $\mathrm{C} 60-\mathrm{C} 61-\mathrm{H} 61$ & 120.5 \\
\hline $\mathrm{C} 20-\mathrm{C} 21-\mathrm{C} 16$ & $122.8(2)$ & $\mathrm{C} 61-\mathrm{C} 62-\mathrm{C} 63$ & $120.1(2)$ \\
\hline $\mathrm{C} 20-\mathrm{C} 21-\mathrm{H} 21$ & 118.6 & $\mathrm{C} 61-\mathrm{C} 62-\mathrm{H} 62$ & 120.0 \\
\hline $\mathrm{C} 16-\mathrm{C} 21-\mathrm{H} 21$ & 118.6 & $\mathrm{C} 63-\mathrm{C} 62-\mathrm{H} 62$ & 120.0 \\
\hline $\mathrm{C} 27-\mathrm{C} 22-\mathrm{C} 23$ & $114.8(2)$ & $\mathrm{C} 62-\mathrm{C} 63-\mathrm{C} 58$ & $123.1(2)$ \\
\hline $\mathrm{C} 27-\mathrm{C} 22-\mathrm{B} 1$ & $124.9(2)$ & $\mathrm{C} 62-\mathrm{C} 63-\mathrm{H} 63$ & 118.5 \\
\hline $\mathrm{C} 23-\mathrm{C} 22-\mathrm{B} 1$ & $120.40(19)$ & $\mathrm{C} 58-\mathrm{C} 63-\mathrm{H} 63$ & 118.5 \\
\hline $\mathrm{C} 5-\mathrm{N} 1-\mathrm{C} 1-\mathrm{N} 2$ & $-154.0(2)$ & $\mathrm{B} 1-\mathrm{C} 28-\mathrm{C} 33-\mathrm{C} 32$ & $177.3(2)$ \\
\hline
\end{tabular}




\begin{tabular}{|c|c|c|c|}
\hline $\mathrm{C} 4-\mathrm{N} 1-\mathrm{C} 1-\mathrm{N} 2$ & $24.1(3)$ & $\mathrm{C} 16-\mathrm{B} 1-\mathrm{C} 34-\mathrm{C} 39$ & $-3.6(3)$ \\
\hline $\mathrm{C} 5-\mathrm{N} 1-\mathrm{C} 1-\mathrm{N} 5$ & $26.5(3)$ & $\mathrm{C} 28-\mathrm{B} 1-\mathrm{C} 34-\mathrm{C} 39$ & $-122.2(2)$ \\
\hline $\mathrm{C} 4-\mathrm{N} 1-\mathrm{C} 1-\mathrm{N} 5$ & $-155.43(19)$ & $\mathrm{C} 22-\mathrm{B} 1-\mathrm{C} 34-\mathrm{C} 39$ & $116.0(2)$ \\
\hline $\mathrm{C} 2-\mathrm{N} 2-\mathrm{C} 1-\mathrm{N} 1$ & $-149.4(2)$ & $\mathrm{C} 16-\mathrm{B} 1-\mathrm{C} 34-\mathrm{C} 35$ & $172.12(19)$ \\
\hline $\mathrm{C} 3-\mathrm{N} 2-\mathrm{C} 1-\mathrm{N} 1$ & $34.7(3)$ & $\mathrm{C} 28-\mathrm{B} 1-\mathrm{C} 34-\mathrm{C} 35$ & $53.6(3)$ \\
\hline $\mathrm{C} 2-\mathrm{N} 2-\mathrm{C} 1-\mathrm{N} 5$ & $30.1(3)$ & $\mathrm{C} 22-\mathrm{B} 1-\mathrm{C} 34-\mathrm{C} 35$ & $-68.3(2)$ \\
\hline $\mathrm{C} 3-\mathrm{N} 2-\mathrm{C} 1-\mathrm{N} 5$ & $-145.8(2)$ & $\mathrm{C} 39-\mathrm{C} 34-\mathrm{C} 35-\mathrm{C} 36$ & $-0.9(3)$ \\
\hline $\mathrm{C} 6-\mathrm{N} 5-\mathrm{C} 1-\mathrm{N} 1$ & $-138.3(2)$ & $\mathrm{B} 1-\mathrm{C} 34-\mathrm{C} 35-\mathrm{C} 36$ & $-177.11(19)$ \\
\hline $\mathrm{C} 11-\mathrm{N} 5-\mathrm{C} 1-\mathrm{N} 1$ & $48.8(3)$ & $\mathrm{C} 34-\mathrm{C} 35-\mathrm{C} 36-\mathrm{C} 37$ & $-0.1(3)$ \\
\hline $\mathrm{C} 6-\mathrm{N} 5-\mathrm{C} 1-\mathrm{N} 2$ & $42.1(3)$ & $\mathrm{C} 35-\mathrm{C} 36-\mathrm{C} 37-\mathrm{C} 38$ & $0.7(3)$ \\
\hline $\mathrm{C} 11-\mathrm{N} 5-\mathrm{C} 1-\mathrm{N} 2$ & $-130.7(2)$ & $\mathrm{C} 36-\mathrm{C} 37-\mathrm{C} 38-\mathrm{C} 39$ & $-0.4(3)$ \\
\hline $\mathrm{C} 8-\mathrm{N} 3-\mathrm{C} 6-\mathrm{N} 4$ & $-147.8(2)$ & $\mathrm{C} 37-\mathrm{C} 38-\mathrm{C} 39-\mathrm{C} 34$ & $-0.7(3)$ \\
\hline $\mathrm{C} 7-\mathrm{N} 3-\mathrm{C} 6-\mathrm{N} 4$ & $36.5(3)$ & $\mathrm{C} 35-\mathrm{C} 34-\mathrm{C} 39-\mathrm{C} 38$ & $1.3(3)$ \\
\hline $\mathrm{C} 8-\mathrm{N} 3-\mathrm{C} 6-\mathrm{N} 5$ & $30.7(3)$ & $\mathrm{B} 1-\mathrm{C} 34-\mathrm{C} 39-\mathrm{C} 38$ & $177.3(2)$ \\
\hline $\mathrm{C} 7-\mathrm{N} 3-\mathrm{C} 6-\mathrm{N} 5$ & $-145.0(2)$ & $\mathrm{C} 52-\mathrm{B} 2-\mathrm{C} 40-\mathrm{C} 41$ & $26.9(3)$ \\
\hline $\mathrm{C} 9-\mathrm{N} 4-\mathrm{C} 6-\mathrm{N} 3$ & $-151.7(2)$ & $\mathrm{C} 58-\mathrm{B} 2-\mathrm{C} 40-\mathrm{C} 41$ & $-93.1(2)$ \\
\hline $\mathrm{C} 10-\mathrm{N} 4-\mathrm{C} 6-\mathrm{N} 3$ & $27.8(3)$ & $\mathrm{C} 46-\mathrm{B} 2-\mathrm{C} 40-\mathrm{C} 41$ & $145.0(2)$ \\
\hline $\mathrm{C} 9-\mathrm{N} 4-\mathrm{C} 6-\mathrm{N} 5$ & $29.8(3)$ & $\mathrm{C} 52-\mathrm{B} 2-\mathrm{C} 40-\mathrm{C} 45$ & $-157.59(19)$ \\
\hline $\mathrm{C} 10-\mathrm{N} 4-\mathrm{C} 6-\mathrm{N} 5$ & $-150.7(2)$ & $\mathrm{C} 58-\mathrm{B} 2-\mathrm{C} 40-\mathrm{C} 45$ & $82.4(2)$ \\
\hline $\mathrm{C} 1-\mathrm{N} 5-\mathrm{C} 6-\mathrm{N} 3$ & $42.4(3)$ & $\mathrm{C} 46-\mathrm{B} 2-\mathrm{C} 40-\mathrm{C} 45$ & $-39.5(3)$ \\
\hline $\mathrm{C} 11-\mathrm{N} 5-\mathrm{C} 6-\mathrm{N} 3$ & $-145.0(2)$ & $\mathrm{C} 45-\mathrm{C} 40-\mathrm{C} 41-\mathrm{C} 42$ & $-0.4(3)$ \\
\hline $\mathrm{C} 1-\mathrm{N} 5-\mathrm{C} 6-\mathrm{N} 4$ & $-139.1(2)$ & $\mathrm{B} 2-\mathrm{C} 40-\mathrm{C} 41-\mathrm{C} 42$ & $175.3(2)$ \\
\hline $\mathrm{C} 11-\mathrm{N} 5-\mathrm{C} 6-\mathrm{N} 4$ & $33.5(3)$ & $\mathrm{C} 40-\mathrm{C} 41-\mathrm{C} 42-\mathrm{C} 43$ & $0.8(3)$ \\
\hline $\mathrm{C} 6-\mathrm{N} 5-\mathrm{C} 11-\mathrm{C} 12$ & $43.4(3)$ & $\mathrm{C} 41-\mathrm{C} 42-\mathrm{C} 43-\mathrm{C} 44$ & $-0.2(3)$ \\
\hline $\mathrm{C} 1-\mathrm{N} 5-\mathrm{C} 11-\mathrm{C} 12$ & $-144.2(2)$ & $\mathrm{C} 42-\mathrm{C} 43-\mathrm{C} 44-\mathrm{C} 45$ & $-0.8(3)$ \\
\hline $\mathrm{N} 5-\mathrm{C} 11-\mathrm{C} 12-\mathrm{C} 13$ & $78.9(3)$ & $\mathrm{C} 43-\mathrm{C} 44-\mathrm{C} 45-\mathrm{C} 40$ & $1.2(3)$ \\
\hline $\mathrm{C} 15-\mathrm{N} 6-\mathrm{C} 13-\mathrm{C} 12$ & $-71.4(2)$ & $\mathrm{C} 41-\mathrm{C} 40-\mathrm{C} 45-\mathrm{C} 44$ & $-0.6(3)$ \\
\hline $\mathrm{C} 14-\mathrm{N} 6-\mathrm{C} 13-\mathrm{C} 12$ & $168.05(19)$ & $\mathrm{B} 2-\mathrm{C} 40-\mathrm{C} 45-\mathrm{C} 44$ & $-176.4(2)$ \\
\hline $\mathrm{C} 11-\mathrm{C} 12-\mathrm{C} 13-\mathrm{N} 6$ & $-72.2(3)$ & $\mathrm{C} 40-\mathrm{B} 2-\mathrm{C} 46-\mathrm{C} 51$ & $146.5(2)$ \\
\hline $\mathrm{C} 34-\mathrm{B} 1-\mathrm{C} 16-\mathrm{C} 17$ & $-92.8(2)$ & $\mathrm{C} 52-\mathrm{B} 2-\mathrm{C} 46-\mathrm{C} 51$ & $-95.1(2)$ \\
\hline $\mathrm{C} 28-\mathrm{B} 1-\mathrm{C} 16-\mathrm{C} 17$ & $24.5(3)$ & $\mathrm{C} 58-\mathrm{B} 2-\mathrm{C} 46-\mathrm{C} 51$ & $26.2(3)$ \\
\hline $\mathrm{C} 22-\mathrm{B} 1-\mathrm{C} 16-\mathrm{C} 17$ & $145.4(2)$ & $\mathrm{C} 40-\mathrm{B} 2-\mathrm{C} 46-\mathrm{C} 47$ & $-36.0(3)$ \\
\hline $\mathrm{C} 34-\mathrm{B} 1-\mathrm{C} 16-\mathrm{C} 21$ & $84.9(2)$ & $\mathrm{C} 52-\mathrm{B} 2-\mathrm{C} 46-\mathrm{C} 47$ & $82.3(2)$ \\
\hline $\mathrm{C} 28-\mathrm{B} 1-\mathrm{C} 16-\mathrm{C} 21$ & $-157.7(2)$ & $\mathrm{C} 58-\mathrm{B} 2-\mathrm{C} 46-\mathrm{C} 47$ & $-156.35(19)$ \\
\hline $\mathrm{C} 22-\mathrm{B} 1-\mathrm{C} 16-\mathrm{C} 21$ & $-36.9(3)$ & $\mathrm{C} 51-\mathrm{C} 46-\mathrm{C} 47-\mathrm{C} 48$ & $0.4(3)$ \\
\hline $\mathrm{C} 21-\mathrm{C} 16-\mathrm{C} 17-\mathrm{C} 18$ & $0.0(3)$ & $\mathrm{B} 2-\mathrm{C} 46-\mathrm{C} 47-\mathrm{C} 48$ & $-177.3(2)$ \\
\hline $\mathrm{B} 1-\mathrm{C} 16-\mathrm{C} 17-\mathrm{C} 18$ & $177.8(2)$ & $\mathrm{C} 46-\mathrm{C} 47-\mathrm{C} 48-\mathrm{C} 49$ & $0.3(3)$ \\
\hline $\mathrm{C} 16-\mathrm{C} 17-\mathrm{C} 18-\mathrm{C} 19$ & $1.1(4)$ & $\mathrm{C} 47-\mathrm{C} 48-\mathrm{C} 49-\mathrm{C} 50$ & $-0.5(3)$ \\
\hline $\mathrm{C} 17-\mathrm{C} 18-\mathrm{C} 19-\mathrm{C} 20$ & $-1.5(4)$ & $\mathrm{C} 48-\mathrm{C} 49-\mathrm{C} 50-\mathrm{C} 51$ & $-0.1(3)$ \\
\hline $\mathrm{C} 18-\mathrm{C} 19-\mathrm{C} 20-\mathrm{C} 21$ & $0.7(4)$ & $\mathrm{C} 49-\mathrm{C} 50-\mathrm{C} 51-\mathrm{C} 46$ & $0.8(4)$ \\
\hline $\mathrm{C} 19-\mathrm{C} 20-\mathrm{C} 21-\mathrm{C} 16$ & $0.4(4)$ & $\mathrm{C} 47-\mathrm{C} 46-\mathrm{C} 51-\mathrm{C} 50$ & $-1.0(3)$ \\
\hline $\mathrm{C} 17-\mathrm{C} 16-\mathrm{C} 21-\mathrm{C} 20$ & $-0.7(3)$ & $\mathrm{B} 2-\mathrm{C} 46-\mathrm{C} 51-\mathrm{C} 50$ & $176.6(2)$ \\
\hline $\mathrm{B} 1-\mathrm{C} 16-\mathrm{C} 21-\mathrm{C} 20$ & $-178.6(2)$ & $\mathrm{C} 40-\mathrm{B} 2-\mathrm{C} 52-\mathrm{C} 57$ & $-106.9(2)$ \\
\hline $\mathrm{C} 34-\mathrm{B} 1-\mathrm{C} 22-\mathrm{C} 27$ & $13.9(3)$ & $\mathrm{C} 58-\mathrm{B} 2-\mathrm{C} 52-\mathrm{C} 57$ & $12.3(3)$ \\
\hline $\mathrm{C} 16-\mathrm{B} 1-\mathrm{C} 22-\mathrm{C} 27$ & $134.4(2)$ & $\mathrm{C} 46-\mathrm{B} 2-\mathrm{C} 52-\mathrm{C} 57$ & $134.5(2)$ \\
\hline $\mathrm{C} 28-\mathrm{B} 1-\mathrm{C} 22-\mathrm{C} 27$ & $-105.9(2)$ & $\mathrm{C} 40-\mathrm{B} 2-\mathrm{C} 52-\mathrm{C} 53$ & $69.0(3)$ \\
\hline $\mathrm{C} 34-\mathrm{B} 1-\mathrm{C} 22-\mathrm{C} 23$ & $-165.91(19)$ & $\mathrm{C} 58-\mathrm{B} 2-\mathrm{C} 52-\mathrm{C} 53$ & $-171.79(19)$ \\
\hline $\mathrm{C} 16-\mathrm{B} 1-\mathrm{C} 22-\mathrm{C} 23$ & $-45.4(3)$ & $\mathrm{C} 46-\mathrm{B} 2-\mathrm{C} 52-\mathrm{C} 53$ & $-49.7(3)$ \\
\hline
\end{tabular}




$\begin{array}{ll}\mathrm{C} 28-\mathrm{B} 1-\mathrm{C} 22-\mathrm{C} 23 & 74.3(2) \\ \mathrm{C} 27-\mathrm{C} 22-\mathrm{C} 23-\mathrm{C} 24 & 1.3(3) \\ \mathrm{B} 1-\mathrm{C} 22-\mathrm{C} 23-\mathrm{C} 24 & -178.9(2) \\ \mathrm{C} 22-\mathrm{C} 23-\mathrm{C} 24-\mathrm{C} 25 & -0.5(3) \\ \mathrm{C} 23-\mathrm{C} 24-\mathrm{C} 25-\mathrm{C} 26 & -0.5(3) \\ \mathrm{C} 24-\mathrm{C} 25-\mathrm{C} 26-\mathrm{C} 27 & 0.7(3) \\ \mathrm{C} 25-\mathrm{C} 26-\mathrm{C} 27-\mathrm{C} 22 & 0.1(3) \\ \mathrm{C} 23-\mathrm{C} 22-\mathrm{C} 27-\mathrm{C} 26 & -1.1(3) \\ \mathrm{B} 1-\mathrm{C} 22-\mathrm{C} 27-\mathrm{C} 26 & 179.1(2) \\ \mathrm{C} 34-\mathrm{B} 1-\mathrm{C} 28-\mathrm{C} 29 & -152.8(2) \\ \mathrm{C} 16-\mathrm{B} 1-\mathrm{C} 28-\mathrm{C} 29 & 88.5(2) \\ \mathrm{C} 22-\mathrm{B} 1-\mathrm{C} 28-\mathrm{C} 29 & -30.5(3) \\ \mathrm{C} 34-\mathrm{B} 1-\mathrm{C} 28-\mathrm{C} 33 & 30.1(3) \\ \mathrm{C} 16-\mathrm{B} 1-\mathrm{C} 28-\mathrm{C} 33 & -88.6(2) \\ \mathrm{C} 22-\mathrm{B} 1-\mathrm{C} 28-\mathrm{C} 33 & 152.4(2) \\ \mathrm{C} 33-\mathrm{C} 28-\mathrm{C} 29-\mathrm{C} 30 & -0.2(3) \\ \mathrm{B} 1-\mathrm{C} 28-\mathrm{C} 29-\mathrm{C} 30 & -177.4(2) \\ \mathrm{C} 28-\mathrm{C} 29-\mathrm{C} 30-\mathrm{C} 31 & -0.1(3) \\ \mathrm{C} 29-\mathrm{C} 30-\mathrm{C} 31-\mathrm{C} 32 & 0.6(3) \\ \mathrm{C} 30-\mathrm{C} 31-\mathrm{C} 32-\mathrm{C} 33 & -0.7(3) \\ \mathrm{C} 31-\mathrm{C} 32-\mathrm{C} 33-\mathrm{C} 28 & 0.5(3) \\ \mathrm{C} 29-\mathrm{C} 28-\mathrm{C} 33-\mathrm{C} 32 & 0.0(3)\end{array}$

$$
\begin{array}{ll}
\mathrm{C} 57-\mathrm{C} 52-\mathrm{C} 53-\mathrm{C} 54 & 1.4(3) \\
\mathrm{B} 2-\mathrm{C} 52-\mathrm{C} 53-\mathrm{C} 54 & -174.8(2) \\
\mathrm{C} 52-\mathrm{C} 53-\mathrm{C} 54-\mathrm{C} 55 & -0.2(3) \\
\mathrm{C} 53-\mathrm{C} 54-\mathrm{C} 55-\mathrm{C} 56 & -1.2(3) \\
\mathrm{C} 54-\mathrm{C} 55-\mathrm{C} 56-\mathrm{C} 57 & 1.3(3) \\
\mathrm{C} 55-\mathrm{C} 56-\mathrm{C} 57-\mathrm{C} 52 & 0.0(4) \\
\mathrm{C} 53-\mathrm{C} 52-\mathrm{C} 57-\mathrm{C} 56 & -1.3(3) \\
\mathrm{B} 2-\mathrm{C} 52-\mathrm{C} 57-\mathrm{C} 56 & 174.8(2) \\
\mathrm{C} 40-\mathrm{B} 2-\mathrm{C} 58-\mathrm{C} 63 & -5.3(3) \\
\mathrm{C} 52-\mathrm{B} 2-\mathrm{C} 58-\mathrm{C} 63 & -124.4(2) \\
\mathrm{C} 46-\mathrm{B} 2-\mathrm{C} 58-\mathrm{C} 63 & 115.2(2) \\
\mathrm{C} 40-\mathrm{B} 2-\mathrm{C} 58-\mathrm{C} 59 & 173.59(19) \\
\mathrm{C} 52-\mathrm{B} 2-\mathrm{C} 58-\mathrm{C} 59 & 54.4(3) \\
\mathrm{C} 46-\mathrm{B} 2-\mathrm{C} 58-\mathrm{C} 59 & -65.9(3) \\
\text { C63-C58-C59-C60 } & -1.6(3) \\
\mathrm{B} 2-\mathrm{C} 58-\mathrm{C} 59-\mathrm{C} 60 & 179.4(2) \\
\text { C58-C59-C60-C61 } & 0.5(3) \\
\text { C59-C60-C61-C62 } & 0.8(3) \\
\text { C60-C61-C62-C63 } & -0.9(3) \\
\text { C61-C62-C63-C58 } & -0.3(3) \\
\text { C59-C58-C63-C62 } & 1.5(3) \\
\text { B2-C58-C63-C62 } & -179.5(2)
\end{array}
$$$$
1.4 \text { (3) }
$$$$
-0.2 \text { (3) }
$$$$
-1.2(3)
$$$$
1.3(3)
$$$$
0.0 \text { (4) }
$$$$
-1.3(3)
$$$$
174.8 \text { (2) }
$$$$
-5.3(3)
$$$$
-124.4(2)
$$$$
115.2(2)
$$$$
173.59(19)
$$$$
54.4 \text { (3) }
$$$$
-65.9(3)
$$$$
-1.6(3)
$$$$
179.4 \text { (2) }
$$$$
0.5 \text { (3) }
$$$$
0.8 \text { (3) }
$$$$
-0.9 \text { (3) }
$$$$
-0.3 \text { (3) }
$$$$
1.5 \text { (3) }
$$$$
-179.5(2)
$$

Hydrogen-bond geometry $\left(\AA,{ }^{\circ}\right)$

$C g 1, C g 2$ and $C g 3$ are the centroids of the $\mathrm{C} 22-\mathrm{C} 27, \mathrm{C} 28-\mathrm{C} 33$ and $\mathrm{C} 58-\mathrm{C} 63$ rings, respectively.

\begin{tabular}{lllll}
\hline$D-\mathrm{H} \cdots A$ & $D-\mathrm{H}$ & $\mathrm{H} \cdots A$ & $D \cdots A$ & $D-\mathrm{H} \cdots A$ \\
\hline $\mathrm{C} 4-\mathrm{H} 4 B \cdots C g 1$ & 0.98 & 2.63 & $3.597(2)$ & 171 \\
$\mathrm{C} 4-\mathrm{H} 4 A \cdots C g 3$ & 0.98 & 2.64 & $3.580(2)$ & 161 \\
$\mathrm{C} 11-\mathrm{H} 11 A \cdots C g 2$ & 0.99 & 2.94 & $3.500(2)$ & 117 \\
$\mathrm{C} 13-\mathrm{H} 13 B \cdots C g 3$ & 0.99 & 2.97 & $3.950(2)$ & 170 \\
\hline
\end{tabular}

